\title{
A Dynamical Initialization Scheme for Tropical Cyclones under the Influence of Terrain
}

\author{
HAO-YAN LIU \\ State Key Laboratory of Severe Weather, Chinese Academy of Meteorological Sciences, China Meteorological \\ Administration, Beijing, China \\ YUQING WANG \\ State Key Laboratory of Severe Weather, Chinese Academy of Meteorological Sciences, China Meteorological \\ Administration, Beijing, China, and International Pacific Research Center, and Department of Atmospheric Sciences, \\ School of Ocean and Earth Science and Technology, University of Hawai'i at Mānoa, Honolulu, Hawaii

\section{JING XU AND YIHONG DUAN} \\ State Key Laboratory of Severe Weather, Chinese Academy of Meteorological Sciences, China Meteorological \\ Administration, Beijing, China
}

(Manuscript received 20 September 2017, in final form 15 February 2018)

\begin{abstract}
This study extends an earlier dynamical initialization (DI) scheme for tropical cyclones (TCs) to situations under the influence of terrain. When any terrain lower than $1 \mathrm{~km}$ exists between 150 and $450 \mathrm{~km}$ from the TC center, topographic variables are defined and a filtering algorithm is used to remove noise due to the presence of terrain before the vortex separation is conducted. When any terrain higher than $1 \mathrm{~km}$ exists between 150 and $300 \mathrm{~km}$ from the TC center, or the TC center is within $150 \mathrm{~km}$ of land, a semi-idealized integration without the terrain is conducted to spin up an axisymmetric TC vortex before the inclusion of the terrain and the merging of the TC vortex with the large-scale analysis field. In addition, a procedure for the vortex size/intensity adjustment is introduced to reduce the initial errors before the forecast run. Two sets of hindcasts, one without (CTRL run) and one with the new DI scheme (DI run), are conducted for nine TCs affected by terrain over the western North Pacific in 2015. Results show that the new DI scheme largely reduces the initial position and intensity errors. The 72-h position errors and the intensity errors up to the 36 - $h$ forecasts are smaller in DI runs than in CTRL runs and smaller than those from the HWRF forecasts for the same TCs as well. The new DI scheme is also shown to produce the TC inner-core structure and rainbands more consistent with satellite and radar observations.
\end{abstract}

\section{Introduction}

The initial conditions of tropical cyclones (TCs) in numerical models benefit from various initialization approaches. One approach is to insert a bogus vortex constructed based on empirical functions for the axisymmetric tangential wind profile and the balanced pressure distribution into the analysis field (Holland 1980; Kwon and Cheong 2010; Rappin et al. 2013). This method is low cost computationally, but the vortex customized is not necessarily consistent with the forecast model. Based on bogus vortex and variational data assimilation techniques, the bogus data assimilation

Corresponding author: Prof. Yuqing Wang, yuqing@hawaii.edu
(BDA; Zou and Xiao 2000; Zhang et al. 2012) was proposed to generate a vortex closer to the observed intensity and structure and dynamically more consistent with the forecast model. Another TC initialization method is the so-called dynamical initialization (DI; Kurihara et al. 1993; Nguyen and Chen 2011; Cha and Wang 2013; Hendricks et al. 2013; Liu and Tan 2016), in which a three-dimensional vortex is generated in an idealized or real-case model run to replace the weak TC vortex in the initial conditions of a numerical forecast model. The TC vortex constructed by a DI scheme can also include the moisture and cloud microphysical fields. The initial conditions improved by this method are more consistent with the prediction model but are computationally expensive. These methods have been shown to 
considerably improve TC track and intensity forecasts in previous studies.

Recently, Cha and Wang (2013, hereafter CW13) introduced a new TC DI scheme (hereafter the CW13 scheme) to spin up a TC vortex using the Weather Research and Forecasting (WRF) Model. In the CW13 scheme, three steps are taken, including 1) vortex separation, 2) cycle runs with large-scale spectral nudging, and 3) TC relocation before the forecast run. For vortex separation, the TC axisymmetric, asymmetric, and environmental components are obtained based on local three-point smoothing and the azimuthal mean (CW13). The TC axisymmetric component is then spun up by several 6 -h integrations initialized at $6 \mathrm{~h}$ before the forecast run in the WRF Model, and the spunup new TC vortex is used as the initial conditions for the next cycle run if the intensity is weaker than the real-time reported storm intensity or the forecast run if the intensity is comparable with the real-time reported intensity. Since the initial conditions in the forecast run come from the last cycle run as a warm start, large-scale spectral nudging is employed to reduce the large-scale bias between the global analysis and the cycle run. Spectral nudging is applied to wind and temperature fields for wavelengths larger than $1000 \mathrm{~km}$ with a heightdependent nudging coefficient. A final relocation procedure is applied before the forecast run to ensure that the TC is initialized at the observed position. The CW13 scheme is able to generate realistic TC initial conditions and reduces the initial position and intensity errors remarkably. However, the CW13 scheme is not applicable to cases when a TC is close to terrain. Namely, the DI is skipped if any terrain within a radius of $200 \mathrm{~km}$ from the TC center is higher than $1 \mathrm{~km}$ (Nguyen and Chen 2011; CW13).

When a TC approaches terrain, the TC structure is more complicated than that over the open ocean (Ramsay and Leslie 2008; Liou et al. 2016). The tangential wind (Yang et al. 2008) and the eyewall structure (Yu and Tsai 2010) could become highly asymmetric under the influence of topographic interaction. High orography, such as the Central Mountain Range (CMR) in Taiwan, might induce a cyclonic vortex on the leeward side (Wu 2001; Yeh et al. 2012). Wang (1980) and Shieh et al. (1998) found that the secondary cyclonic vortex or low pressure center appearing on the leeward side of the CMR might cause discontinuity in the TC track when the storm crossed the island (Wang 1980; Yeh and Elsberry 1993; Lin et al. 2005, 2006). In addition to the difficulty in the initialization of a TC near terrain, because of the distortion of the TC circulation, the definition of the storm center is even problematic in some circumstances, particularly for the case when the storm weakens rapidly under the influence of terrain.
Although DI is an effective way to reduce the position and intensity errors in the initial conditions for a TC in a numerical weather prediction model, the DI for a TC under the influence of terrain has not been explored in the literature. In this study, the DI scheme developed by CW13 is extended to the situation under the influence of terrain so that the initial conditions and forecasts for TCs by numerical models can be improved. The rest of the paper is organized as follows. The new DI scheme is introduced in section 2. Section 3 describes the numerical model and experimental design. In section 4, two TC case studies are discussed. Forecast skill for nine TCs affected by terrain over the western North Pacific in 2015 is evaluated in section 5 . A brief summary is given in the last section.

\section{Description of the new DI scheme under the influence of terrain}

A key to the CW13 DI scheme is to spin up only the axisymmetric component of a TC vortex through cycle runs. When a TC approaches terrain, the axisymmetric structure may not be easily determined. Furthermore, once a TC moves across terrain, its circulation may be largely distorted with considerable mesoscale and smallscale structures. In these cases, not only the axisymmetric TC structure but also the TC center is hard to determine, greatly limiting the use of the TC DI previously developed. To fill this gap, in this study, we propose a new DI scheme for a TC that moves close by or across terrain. To extend the DI scheme proposed in CW13 to the situation under the influence of terrain, three orographic situations are considered where the distance between the TC center and the terrain is 1) less than $450 \mathrm{~km}, 2$ ) less than $300 \mathrm{~km}$ with the maximum height of the terrain greater than $1 \mathrm{~km}$, and 3) less than $150 \mathrm{~km}$ when the TC inner core is partially over the terrain or land. In addition, the vortex size adjustment introduced in Liu and Tan (2016), which reshapes the TC initial structure of tangential wind to fit the radius of maximum wind (RMW) in the Joint Typhoon Warning Center (JTWC) real-time TC report, has also been used in the new DI scheme. The vortex-scale adjustment is a linear interpolation designed to rescale the radius of maximum axisymmetric tangential wind before each cycle run.

Furthermore, since the TC intensity after the last cycle run might not be exactly equal to (a bit stronger or weaker) the real-time reported storm intensity (hereafter OBS), we introduce the vortex intensity adjustment to reduce the initial intensity difference in the forecast run. Before the forecast run (hindcast), we calculate the bias of the maximum surface wind speed between the initial conditions of the forecast run and OBS. For the situation where the absolute value of the bias is larger than $1 \mathrm{~m} \mathrm{~s}^{-1}$, the vortex component of the winds and perturbation 


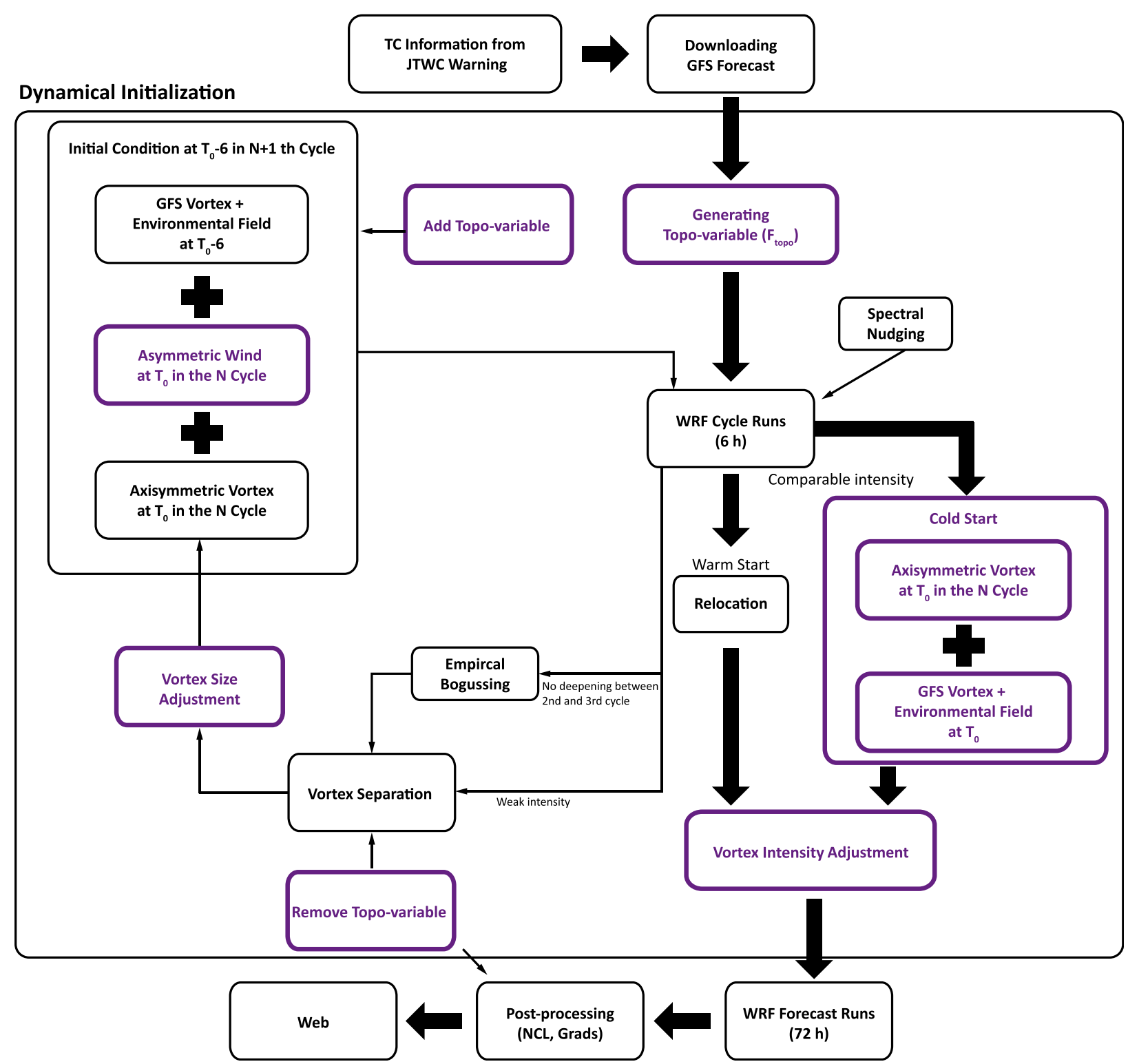

FIG. 1. Flowchart for the new DI scheme. Components shown with a black font correspond to the original CW13 scheme, and those in purple correspond to the new DI scheme (see text for details).

pressure in the initial conditions will be multiplied by a coefficient, which is defined as the ratio of the maximum surface wind in the real-time report to that in the initial conditions of the forecast run. Compared with $\mathrm{CW} 13$, the inclusion of the vortex intensity adjustment can further reduce the TC initial intensity error to less than $1 \mathrm{~m} \mathrm{~s}^{-1}$ (see Figs. 14 and 15 in section 5).

Figure 1 shows the flowchart of the new DI scheme, in which the black parts are the same as those in CW13, and the purple parts are new components that represent the special procedures applied to TCs affected by terrain. As shown in Fig. 1, topographic variables are generated based on the global analysis. A scalar variable can be decomposed into two parts as follows:

$$
F=F_{\text {global }}+F_{\sigma}
$$

where $F$ is the scalar variable from the WRF Model initial conditions, which is interpolated from the global analysis $F_{\text {global }}$ excluding the winds that remain unchanged because winds are often not adjusted for the existence of terrain when the global analysis is performed. Here, $F_{\sigma}$ is the difference between the total fields from the WRF Model initial conditions, which are 
(a)

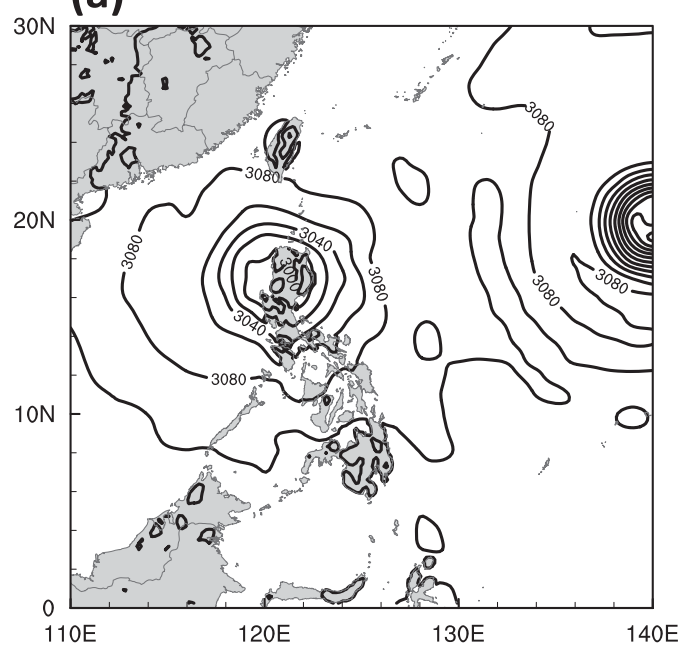

(c)

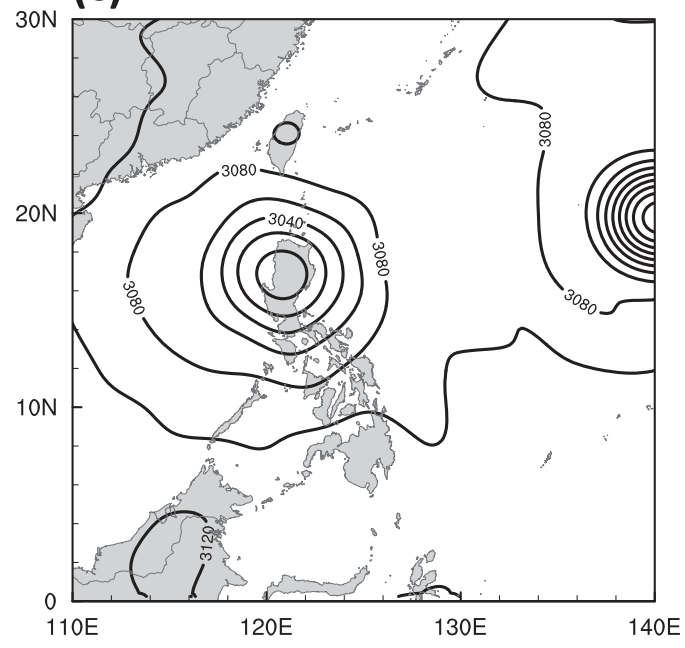

(b)

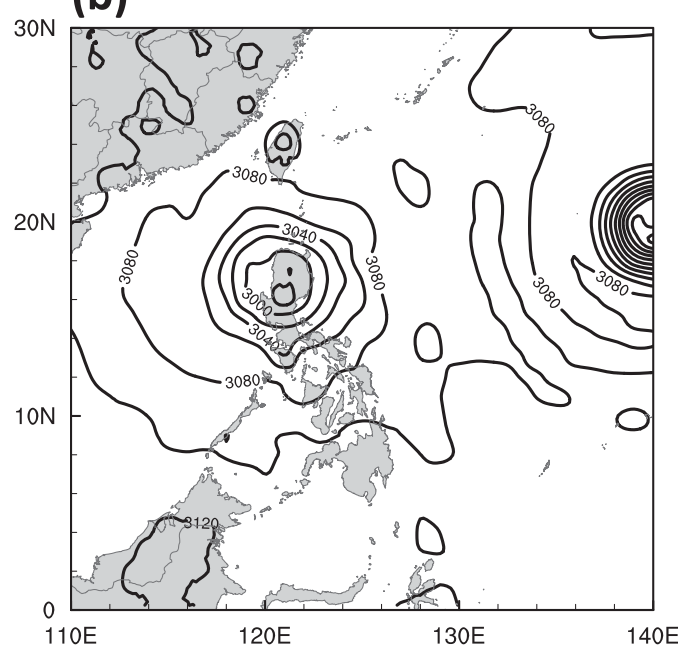

(d)

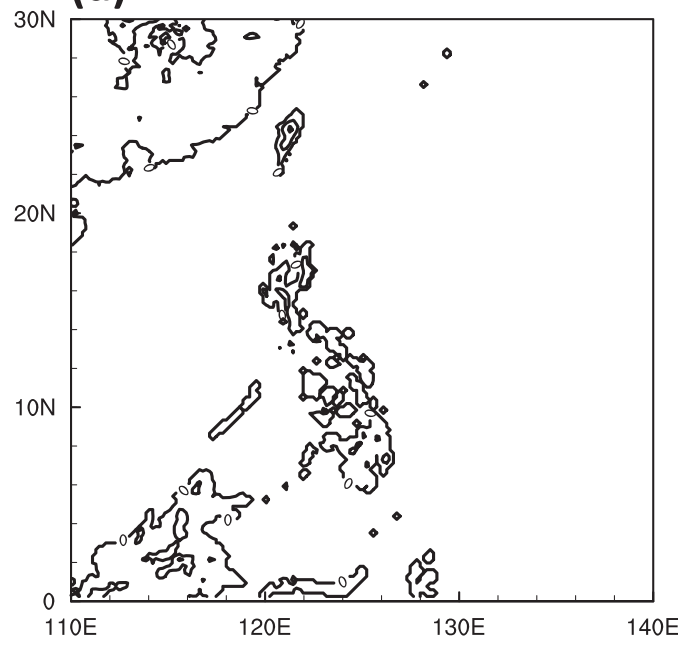

FIG. 2. Geopotential height at $700 \mathrm{hPa}$ (gpm; 20-gpm interval) at $1200 \mathrm{UTC} 18$ Oct 2015 for Supertyphoon Koppu (2015) near Luzon from the WRF grids interpolated from the coarse-resolution FNL dataset (a) with and (b) without terrain. Shown are the corresponding (c) filtered and (d) filtered-out fields.

interpolated from the global analysis in pressure coordinates to the high horizontal resolution WRF Model in $\sigma$ coordinates, with terrain (Fig. 2a) and without terrain (Fig. 2b). The value of $F_{\text {global }}$ is assumed to be the same as that given in the global analysis since the global analysis fields are often given in pressure coordinates without the explicit representation of terrain.

In practice, we found that the presence of terrain often causes some noise in the WRF Model initial conditions interpolated from the global analysis. An example of the geopotential height at $700 \mathrm{hPa}$ is given in Fig. 2a, which shows some noise near the topography over the islands of Taiwan and Luzon. In the initial conditions without topography included, the geopotential height $\left(F_{\text {global }}\right)$ is much smoother, but some noise still exists in regions with topography (Fig. 2b). This noise is mainly related to the coarse structure of the terrain implicitly involved in the global analysis. To remove the noise unrelated to the TC vortex, a zonal and meridional filtering algorithm is used to calculate the disturbance component referred as the noise. The algorithm is based on the local three-point smoothing operator as used in Nguyen and Chen (2011), with the smoothing coefficient $K_{m}$ being fixed as

$$
K_{m}=\left[\frac{1}{2}\left(1-\cos \frac{2 \pi}{m}\right)^{-1}\right], \quad m=2 .
$$

Using the topographic filtering above, $F_{\text {global }}$ can be decomposed into two components as follows: 


$$
F_{\text {global }}=F_{\text {notopo }}+F_{\text {filter }} \text {, }
$$

where $F_{\text {notopo }}$ is the smoothed field after topographic filtering (Fig. 2c), and $F_{\text {filter }}$ is the residual. Note that the application with $K_{m}$ given in Eq. (2) can completely remove all scales with wavelengths less than about $200 \mathrm{~km}$, which is comparable to the orographic size of the islands of Taiwan, Hainan, and Luzon. This is chosen because we tentatively apply this new DI scheme for the western North Pacific, including the South China Sea. After the filtering, the vortex-scale and large-scale components have been well preserved, and the vortex circulation is as smooth as that over the open ocean (Fig. 2c). Finally, we define a topographic variable as

$$
F_{\text {topo }}=F_{\sigma}+F_{\text {filter }},
$$

which is the difference between the total fields in the WRF initial conditions $F$ (Fig. 2a) and the filtered fields $F_{\text {notopo }}$ (Fig. 2c). For reference, we show the field $F_{\text {topo }}$ in Fig. 2d. We can see that the distribution of noise in geopotential height that has been filtered out adheres to the terrain with the horizontal scale generally less than $200 \mathrm{~km}$. As a result, Eq. (1) can be rewritten as

$$
F=F_{\text {notopo }}+F_{\text {topo }} .
$$

Based on the algorithm used to merge the spunup axisymmetric TC component and the analysis (CW13), three situations are considered in the new DI scheme. In the first situation (STN1), a TC is located over the open ocean with the distance between the TC center and the nearest land (hereafter $\Delta x$ ) being beyond $450 \mathrm{~km}$. In the second situation (STN2), $\Delta x$ is between 150 and $450 \mathrm{~km}$, within which the maximum height of the terrain is less than $1 \mathrm{~km}$ (referred to as orographic situation 1 earlier in this section). In the last situation (STN3), $\Delta x$ is less than $300 \mathrm{~km}$, within which the maximum height of the terrain is greater than $1 \mathrm{~km}$ or the terrain is located in the TC inner core when $\Delta x$ is less than $150 \mathrm{~km}$ (referred to as orographic situations 2 and 3 above). The overlap between STN2 and STN3 is considered to be STN3. The areas of the three situations in the western North Pacific are shown in Fig. 3.

Note that the criteria for defining the three situations (STN1, STN2, and STN3) are determined based on the definition of the vortex component used to merge the vortex component with the analysis field in the cycle runs. The region within a radius of $150 \mathrm{~km}$ from the storm center is considered to be the inner core of the targeted TC. If the TC center is within $150 \mathrm{~km}$ from the terrain, the TC inner-core structure will be largely distorted as a result of the orographic interaction. In this situation, cycle runs with no terrain are conducted (see

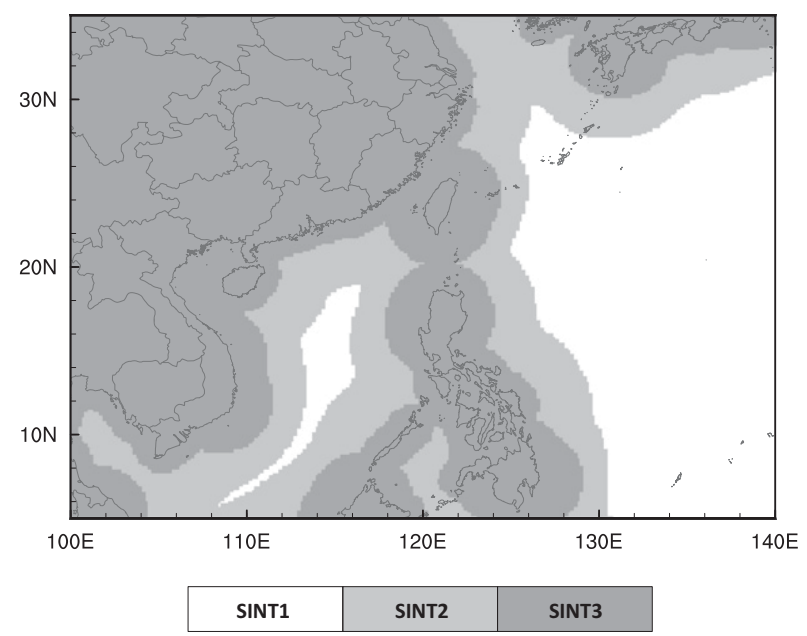

FIG. 3. The areas for the situations of cycle runs in the new DI scheme over the western North Pacific. White areas are for STN1, light gray for STN2, and dark gray for STN3.

below). The region within a radius of $450 \mathrm{~km}$ is considered to be the primary TC circulation that is to be spun up through the cycle runs in the DI. The vortex component and the environmental analysis field are merged with a blended weighting function between 300 and $600 \mathrm{~km}$, as given in CW13 [see their Eq. (9)]. As a result, the use of $450 \mathrm{~km}$ is to ensure a smooth merging of the spunup vortex component and the analysis field after each cycle run. It is a shortcoming to use a fixed radius in the current settings since it is better to vary the blending region based on the size of the targeted TC. This, however, remains an issue for future efforts because no reliable information related to the TC circulation size is available in real time at present. Nevertheless, the application of the current settings still shows the significant improvements, as will be discussed in sections 4 and 5 below and thus is acceptable.

\section{a. TCs over the open ocean}

For situations involving TCs over the open ocean with $\Delta x$ larger than $450 \mathrm{~km}$ (STN1), there is no need to consider the orographic effect in the CW13 scheme, since the scheme spins up the inner part of the TC vortex. In the new DI scheme, all procedures are similar to those described in CW13, except that an adjustment for the size of the initial TC vortex is applied to give a more realistic inner-core structure of the axisymmetric TC vortex after each cycle run, following Liu and Tan (2016). In addition, the asymmetric wind component of the improved vortex at $T_{0}$ during the $N$ th cycle run has also been introduced in the new DI scheme (Fig. 1). Figure 4 compares the initial conditions of the first cycle run and the initial conditions for the forecast run (namely after the last cycle run) for the case of Typhoon 
(a)

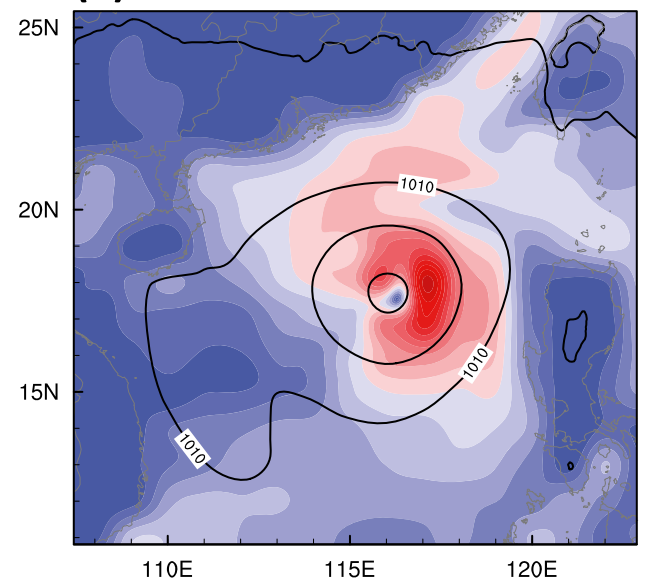

(c)

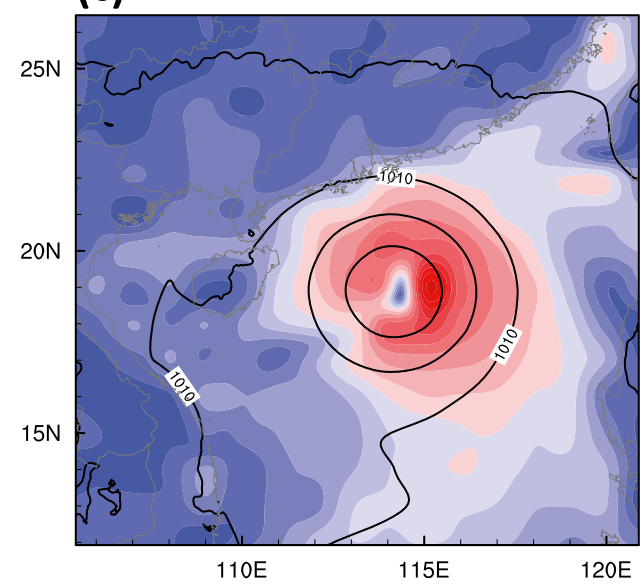

(e)

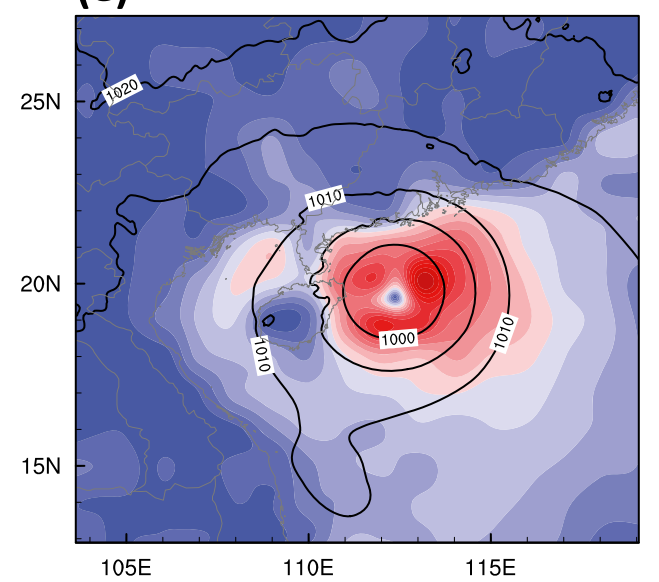

(b)

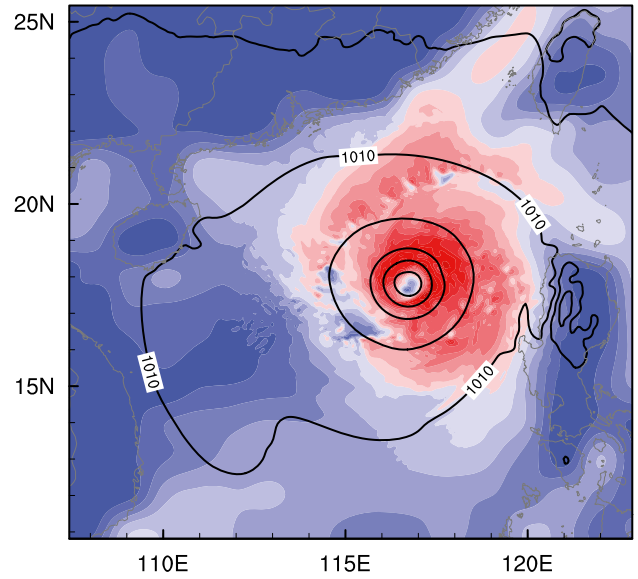

(d)

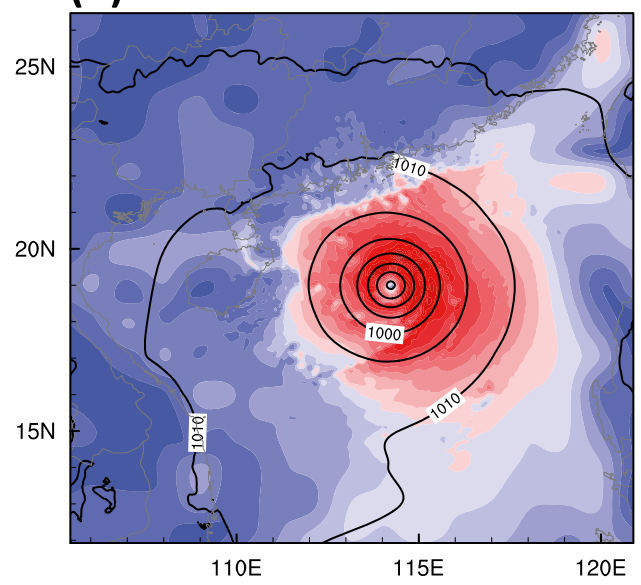

(f)

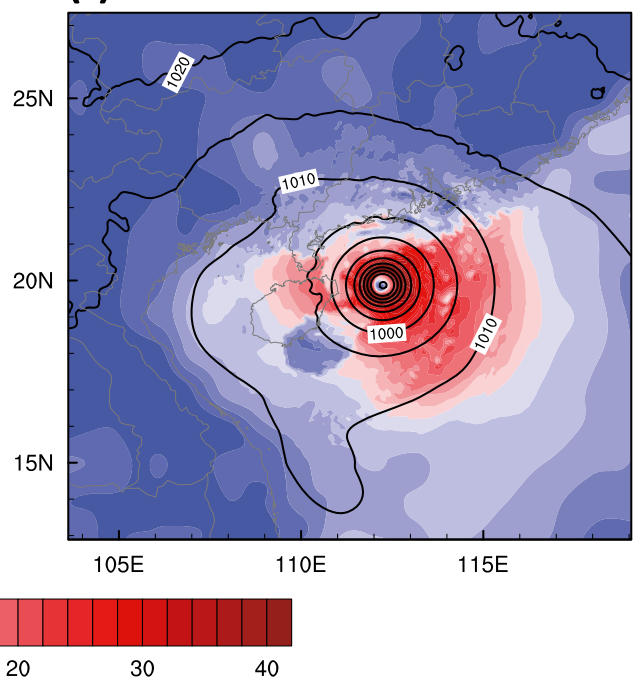

FIG. 4. Sea level pressure (hPa; contours) and 10-m wind speed ( $\mathrm{m} \mathrm{s}^{-1}$; shading) of Typhoon Mujigae (2015) for the initial time $t_{0}-6$ of cycle runs interpolated from the FNL dataset at (a) 1800 UTC 2 Oct, (c) 0600 UTC 3 Oct, and (e) 1800 UTC 3 Oct. (b),(d),(f) As in (a),(c),(e) but for the last cycle runs. 


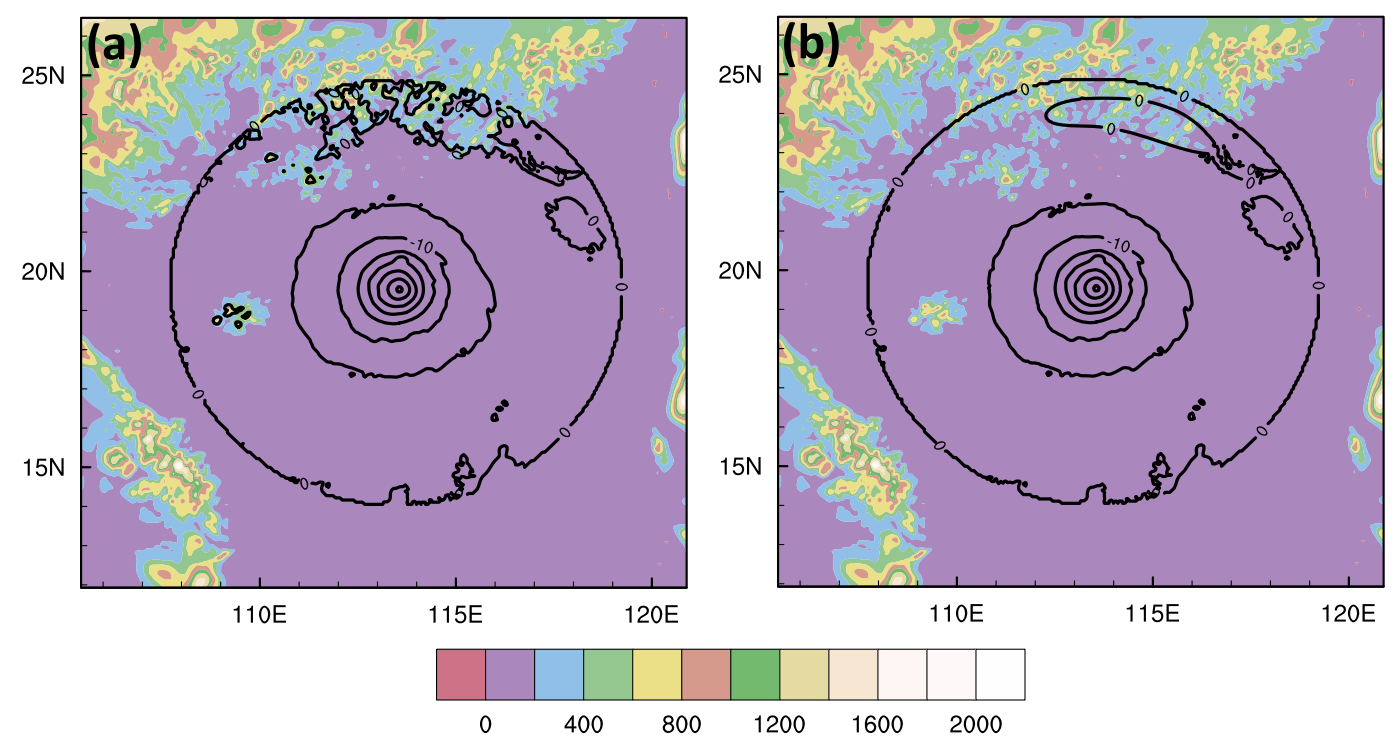

FIG. 5. Perturbation pressure of the vortex component at the lowest sigma level (hPa; contours with 5-hPa intervals) and terrain height (m; shading) for Typhoon Mujigae (2015) at 1200 UTC 3 Oct (a) without and (b) with topographic adjustment.

Mujigae (2015). Both the sea level pressure (SLP) and horizontal wind speed indicate the deepened TC vortex after the last cycle run (Fig. 4b) compared to the first cycle run (Fig. 4a). As a result of the inclusion of asymmetric winds, the horizontal wind at $10-\mathrm{m}$ height shows a more detailed structure. In addition, the vortex intensity adjustment prior to the forecast run briefly described above is also implemented to reduce the initial TC intensity error in the forecast run in the new DI scheme.

\section{b. TCs near low terrain}

When a TC approaches low terrain $(\leq 1 \mathrm{~km})$ with $150 \mathrm{~km}<\Delta x \leq 450 \mathrm{~km}$, a special adjustment is needed to filter the noise related to the orographic effect. For this situation (STN2), the topographic component $F_{\text {topo }}$ should be removed, and the nontopographic component $F_{\text {notopo }}$ should be used in the vortex separation. The difference in the vortex components with and without the above topographic adjustment is shown in Fig. 5. Note that, without the topographic adjustment, the perturbation pressure over high terrain shows some local features that might lead to an unrealistic axisymmetric component calculation (Fig. 5a). With the topographic adjustment, the perturbation pressure of the vortex component appears to be smooth near the terrain, and the axisymmetric component of the TC vortex can be computed as in STN1 (Fig. 5b). After merging both the axisymmetric and asymmetric components of the vortex with the environmental analysis field, $F_{\text {topo }}$ should be added back in to ensure that the orographic forcing is retained in the initial conditions of the forecast run. Figures $4 \mathrm{c}$ and $4 \mathrm{~d}$ show the initial conditions of the first cycle run and the last cycle run. The vortex deepened successfully (Fig. 4d) with a more reasonable vortex structure. Note that the forecast runs from both STN1 and STN2 are warm starts, similar to those in the CW13 scheme.

\section{c. TCs near or over high terrain}

When the TC center moves close to the terrain $(\Delta x \leq 300 \mathrm{~km})$ and the terrain is higher than $1 \mathrm{~km}$, or the TC inner core is located within $150 \mathrm{~km}$ of the terrain $(\Delta x \leq 150 \mathrm{~km})$, semi-idealized (i.e., without topography) cycle runs (STN3) are used to spin up the axisymmetric TC vortex, which is different from either STN1 or STN2. This is because the orography may distort, weaken, or even destroy the TC vortex, and the special adjustment described in section $2 \mathrm{~b}$ above may result in a substantial weakening of the TC after filtering the meso- and small-scale features.

For this situation, the cycle runs are performed as if the TC vortex is over the open ocean first and the land (and terrain) is removed before the cycle runs. As a result, the TC vortex appears to be more axisymmetric after each cycle run since no orographic forcing is included (not shown). The procedures involved in the vortex separation and merger with the environment are similar to those used for TCs over the open ocean as in STN1. Once the vortex intensity is comparable to the best-track data or the real-time reported TC intensity, the axisymmetric vortex component from the semiidealized integration at the forecast time $t_{0}$ is used to replace that in the initial conditions from the global analysis for the forecast run. Therefore, different from STN1 and STN2, the forecast run from STN3 is cold 
started. An example for Typhoon Mujigae (2015) influenced by Hainan Island is given in Fig. $4 \mathrm{f}$ and can be compared with the forecast run without the use of the new DI scheme given in Fig. 4e.

\section{Numerical model and experimental design}

In this study, we used the WRF Model, version 3.8.1 (Skamarock et al.2008), for the cycle runs in the new TC DI scheme and also for the subsequent numerical simulations/hindcasts. The model meshes and physical parameterization schemes were similar to those used in CW13. Three two-way nesting meshes, consisting of $331 \times 301$ (D01), $271 \times 271$ (D02), and $211 \times 211(\mathrm{D} 03)$ grid points with horizontal resolutions of 18,6 , and $2 \mathrm{~km}$, respectively, were configured for the model domain. The outermost mesh (D01) covered the large-scale environment of a TC. The intermediate mesh (D02) and the innermost mesh (D03) covered the primary TC circulation and the inner core of the TC vortex, respectively, and both moved following the model TC. There were 50 vertical levels between the surface and the model top at $10 \mathrm{hPa}$. The time steps of the model integration for the three meshes were 60,20 , and $6.7 \mathrm{~s}$, respectively. All hindcasts were integrated for $72 \mathrm{~h}$ since we mainly focused on cases under the influence of terrain. The model physics included the WRF single-moment 6-class microphysics scheme (WSM6; Hong et al. 2004), Dudhia shortwave radiation scheme (Dudhia 1989), Rapid Radiative Transfer Model (RRTM) longwave radiation scheme (Mlawer et al. 1997), Yonsei University (YSU) planetary boundary layer scheme (Hong et al.2006), and the Noah land surface model (Chen and Dudhia 2001). These schemes were applied for all meshes. The KainFritsch cumulus parameterization scheme (Kain and Fritsch 1990) was applied only to D01. The parameterizations of surface momentum and heat fluxes over the ocean, as well as dissipative heating applicable to strong winds (Davis et al. 2008), were employed with the momentum roughness length from Donelan et al. (2004) and the heat and moisture roughness lengths from Garratt (1992).

The initial and lateral boundary conditions for all experiments were obtained from the National Centers for the Environment Prediction (NCEP) Final (FNL) analyses with $1^{\circ}$ horizontal resolution. Sea surface temperature (SST) was interpolated from the FNL data and fixed in the experiments. The TC best-track data were obtained from JTWC. In the cycle runs of the new DI scheme, only the outer two meshes (D01 and D02) were activated as in CW13 and integrated forward for $6 \mathrm{~h}$ from $6 \mathrm{~h}$ before the initial forecast time $t_{0}-6$ to the initial forecast time $t_{0}$. The model setup for the cycle
TABLE 1. List of TCs in 2015 affected by terrain/land and the number of forecasts for each TC initialized at 0000 and 1200 UTC each day. The forecast initialized at 0000 UTC 14 Dec 2015 was excluded because there was no forecast result available at that time in JTWC-fcst.

\begin{tabular}{llc}
\hline \multicolumn{1}{c}{ TC } & \multicolumn{1}{c}{ Forecast initial time } & No. of forecasts \\
\hline Mekkhala & 1200 UTC 16 Jan-0000 UTC 18 Jan & 4 \\
Noul & 0000 UTC 5 May-1200 UTC 11 May & 14 \\
Kujira & 0000 UTC 22 Jun-0000 UTC 23 Jun & 3 \\
Linfa & 1200 UTC 3 Jul-1200 UTC 6 Jul & 7 \\
Soudelor & 1200 UTC 2 Oct-0000 UTC 8 Aug & 12 \\
Dujuan & 0000 UTC 24 Sep-1200 UTC 28 Sep & 10 \\
Mujigae & 0000 UTC 2 Oct-1200 UTC 4 Oct & 6 \\
Koppu & 0000 UTC 15 Oct-1200 UTC 19 Oct & 10 \\
Melor & 1200 UTC 12 Dec-0000 UTC 15 Dec & 5 \\
Total & & 71 \\
\hline
\end{tabular}

runs was the same as that for the forecast runs except that large-scale spectral nudging was applied in the cycle runs but not in the forecast runs.

Two parallel forecast runs were performed for each set of initial conditions: one with (DI) and one without (CTRL) the use of the new DI scheme. In the CTRL runs, the initial conditions were interpolated directly from the FNL data, and the initial conditions in the DI runs were improved with the use of the new DI scheme described in section 2. We conducted hindcasts for nine TCs that occurred over the western North Pacific during 2015 and were affected by the terrain (see Table 1), so that the strength of the new DI scheme could be demonstrated. In addition, since the new DI scheme includes both the topographic adjustment and the intensity/size adjustment, another set of experiments using the new DI scheme without the vortex intensity/size adjustment (NOADJ) were conducted to isolate the overall contribution of the topographic adjustment alone with the results discussed in section 5. Note that only STN3 cases were run for NOADJ, and all runs including the CTRL and DI runs that were rerun for a comparison were cold started to keep the large-scale environment fields the same in the initial conditions.

\section{Two case studies}

In this section, the performance of the new DI scheme for the initialization of two TCs and the subsequent forecasts, particularly under the influence of terrain, are discussed in detail.

\section{a. Supertyphoon Dujuan}

Supertyphoon Dujuan (2015) formed east of the Philippines over the western North Pacific at 0000 UTC 23 September 2015 and then moved northwestward and passed Taiwan (Fig. 6). The maximum surface 

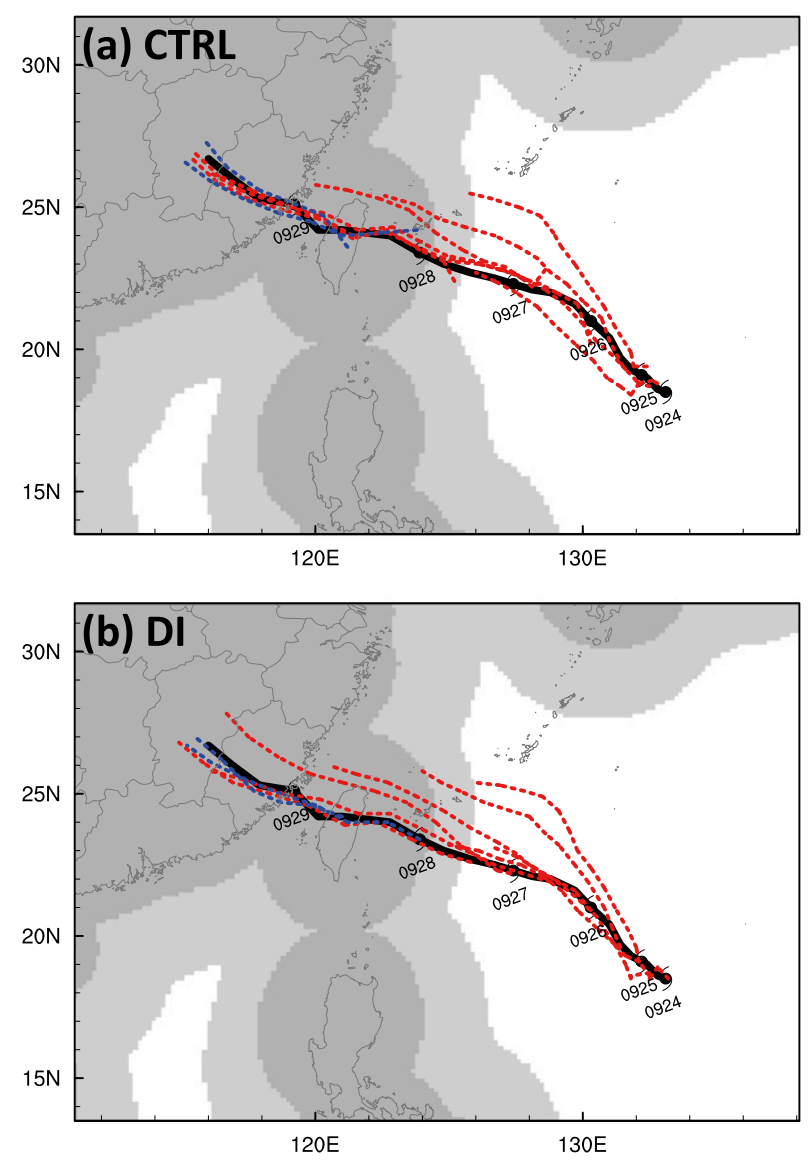

FIG. 6. The simulated and JTWC best-track data (black) tracks for Dujuan (2015) from 0000 UTC 24 Sep to 1800 UTC 29 Sep. The positions are defined as the locations of the MSLP after the topographic adjustment in hindcasts from the (a) CTRL and (b) DI runs. Red dashed lines are referred to as STN1 runs, and blue dashed lines are referred to as STN3 runs. The initial times have a 12-h interval, and the gray areas are the same as those shown in Fig. 3. Note that STN2 is excluded during the 12-h interval simulations of Dujuan.

wind (MSW) was $66.0 \mathrm{~m} \mathrm{~s}^{-1}$ and the minimum central sea level pressure (MSLP) was $926.0 \mathrm{hPa}$ at 1800 UTC 27 September according to the JTWC best-track data. We show the predicted tracks for the CTRL (Fig. 6a, red dashed) and DI runs (Fig. 6b, blue dashed) initialized from 0000 UTC 24 September to 1200 UTC 28 September with 12-h intervals. In CTRL, there were considerable initial position errors (averaged error of $58.9 \mathrm{~km}$ ) compared with the JTWC best-track data. The model tracks deviated to the north except for one forecast initialized at 0000 UTC 24 September. In the following, we mainly focus on the results of the hindcasts under the influence of terrain (STN2 and STN3). The use of the new DI scheme not only reduced the initial position errors, but also improved the 30-h track forecasts, which covered the period from the initialized time
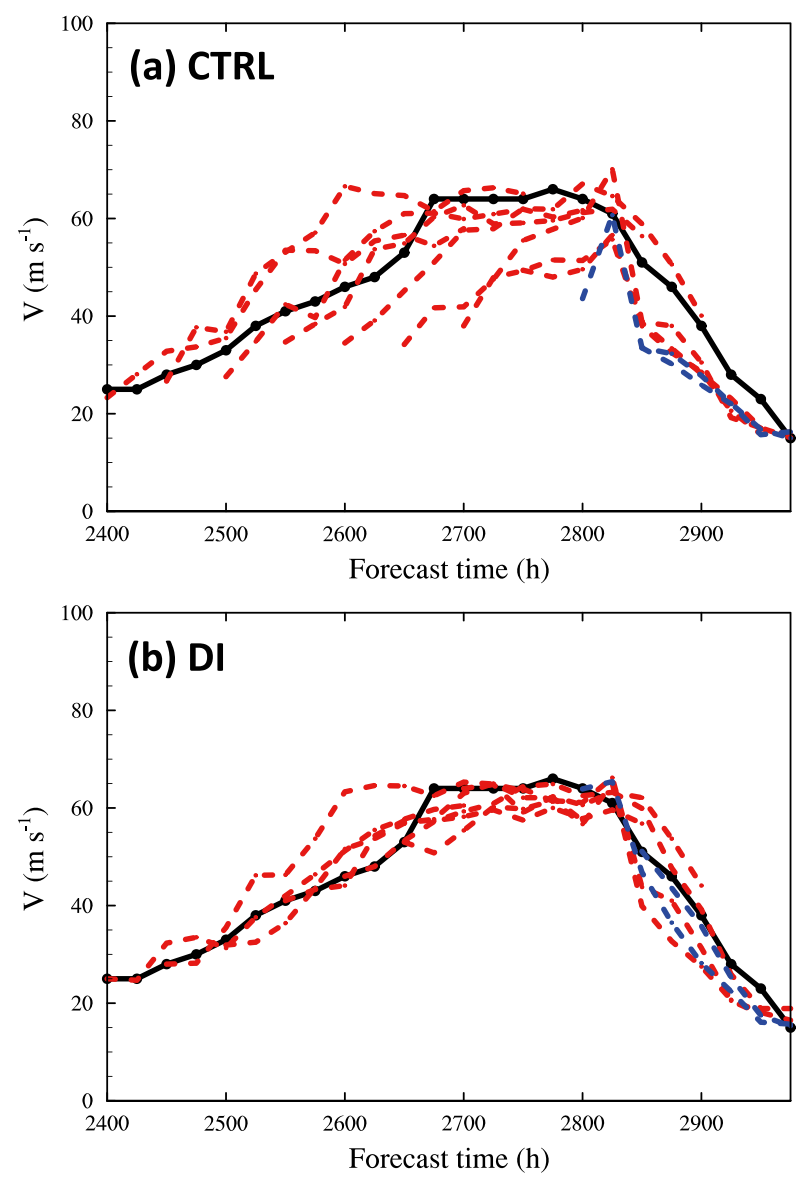

FIG. 7. Temporal evolution of maximum $10-\mathrm{m}$ wind speed $\left(\mathrm{m} \mathrm{s}^{-1}\right)$ of Dujuan (2015) from 0000 UTC 24 Sep to 1800 UTC 29 Sep in the JTWC best-track data (black thick solid line) and in the hindcasts from the (a) CTRL and (b) DI runs as in Fig. 6.

in STN2 and STN3 to the end of the lifetime of Dujuan, with an averaged $30-\mathrm{h}$ position error of $57.3 \mathrm{~km}$ compared with that of $63.9 \mathrm{~km}$ in the CTRL runs.

The JTWC best-track data and modeled 72-h intensity evolutions of Dujuan are shown in Fig. 7. In the CTRL runs, the initial intensities were weaker than the JTWC best-track data except for two forecasts initialized at 0000 and 1200 UTC 24 September. The weak initial intensity in the CTRL runs caused the underpredicted intensity throughout the 72-h forecasts. In comparison with the CTRL runs, the initial intensity errors were largely reduced in the DI runs. The intensity evolution in the DI runs was close to that in the JTWC best-track dataset as well. In the situations directly influenced by terrain (STN2 and STN3), the initial absolute mean intensity error was $0.4 \mathrm{~m} \mathrm{~s}^{-1}$ in the DI runs compared with $17.6 \mathrm{~m} \mathrm{~s}^{-1}$ in the CTRL runs. The mean $30-\mathrm{h}$ absolute intensity error was $3.0 \mathrm{~m} \mathrm{~s}^{-1}$ in the DI runs, which was smaller than that $\left(3.5 \mathrm{~m} \mathrm{~s}^{-1}\right)$ in the CTRL runs. 
(a)

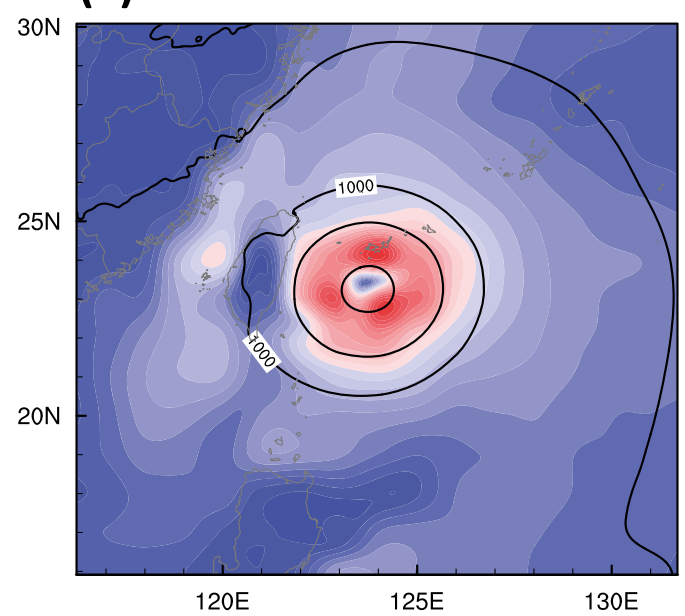

(c)

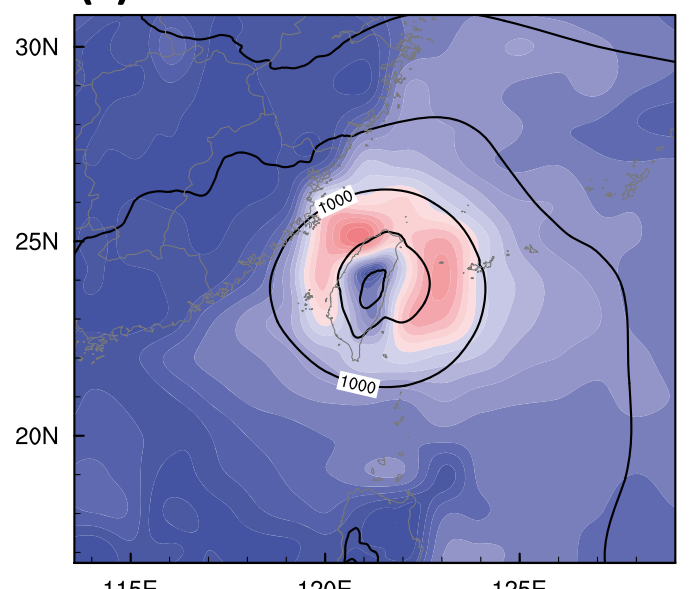

(b)

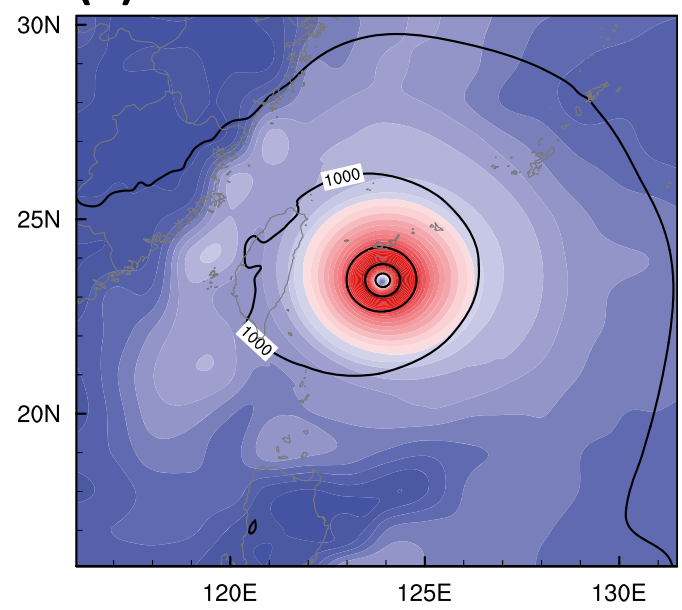

(d)

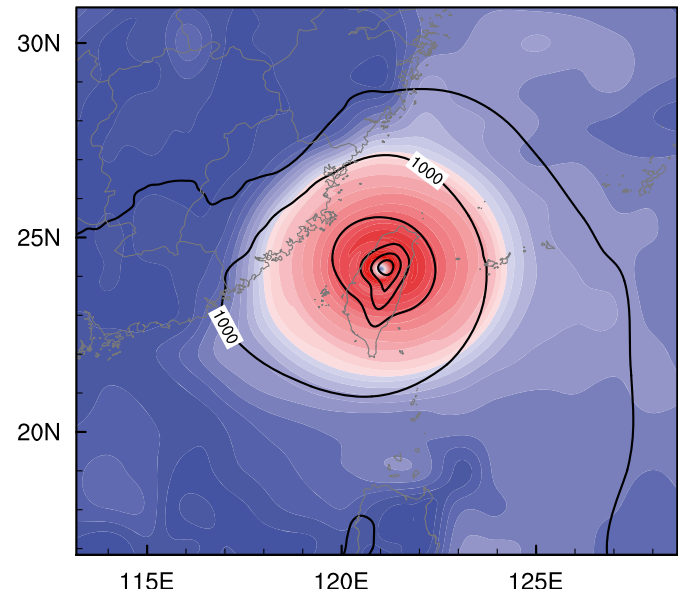

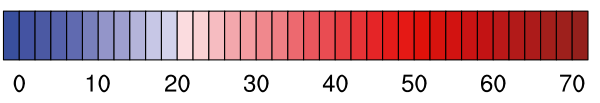

FIG. 8. Sea level pressure (hPa; contours) and 10-m wind speed ( $\mathrm{m} \mathrm{s}^{-1}$; shading) for Supertyphoon Dujuan (2015) at the initial times (a),(b) 0000 and (c),(d) 1200 UTC 28 Sep. Shown are results for the (left) CTRL and (right) DI runs.

Figure 8 shows the initial conditions of Dujuan when it was close to Taiwan at 0000 and 1200 UTC 28 September. Dujuan was located east of Taiwan at 0000 UTC 28 September and was within $150 \mathrm{~km}$ of the east coast of Taiwan. The MSW was $43.6 \mathrm{~m} \mathrm{~s}^{-1}$ in the CTRL run (Fig. 8a), which was weaker than $64.0 \mathrm{~m} \mathrm{~s}^{-1}$ from the JTWC best-track dataset. In the DI run (Fig. 8b), the MSW was $63.9 \mathrm{~m} \mathrm{~s}^{-1}$, which is almost equal to that in the JTWC best-track data, and the MSLP was significantly deeper than that in the CTRL run. Dujuan made landfall over Taiwan $12 \mathrm{~h}$ later. It weakened to $51 \mathrm{~m} \mathrm{~s}^{-1}$ as a result of the orographic effect. The MSW initialized at this time was only $33.5 \mathrm{~m} \mathrm{~s}^{-1}$ in the CTRL run (Fig. 8c). The new DI scheme achieved an intensity of $51.1 \mathrm{~m} \mathrm{~s}^{-1}$ for the storm at this time, almost the same as that in the JTWC best-track dataset. Note that, so far, no DI schemes can deepen a TC vortex when it is located over Taiwan. The new DI scheme developed in this study successfully strengthened Dujuan over Taiwan and also improved the subsequent intensity forecasts.

To demonstrate the improved forecast of storm structure under the influence of terrain with the use of the new DI scheme, we show in Fig. 9 the horizontal TC structure in the CTRL and DI runs at 0000 UTC 29 September when Dujuan was making landfall in Fujian Province. The forecast was initialized at 1200 UTC 28 September when Dujuan was over Taiwan. In the visible satellite image (Fig. 9a), the TC showed active spiral rainbands 
(a)

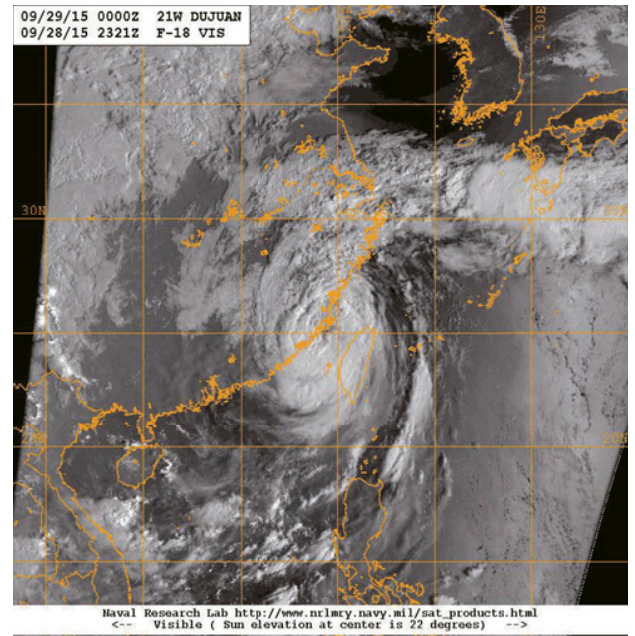

(c)

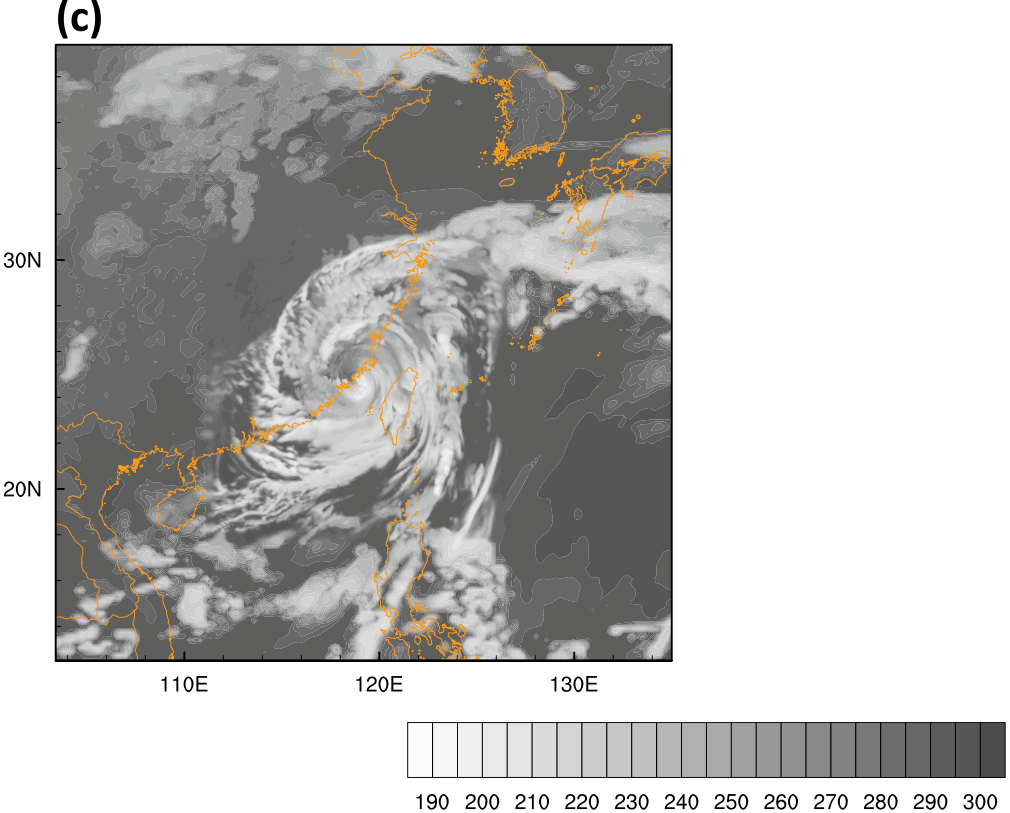

(b)

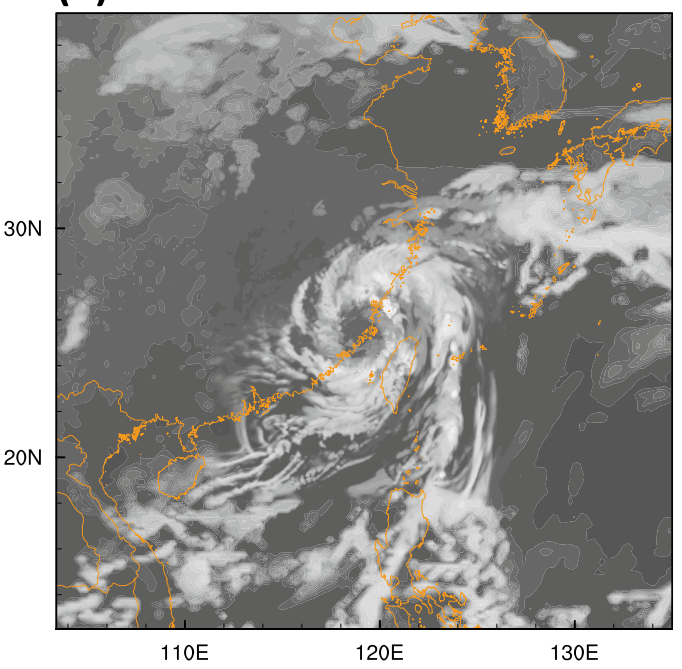

FIG. 9. (a) Visible image from DMSP $5 D-3 F 18$ at 2321 UTC 28 Sep and the horizontal distributions of the simulated blackbody temperature (K) at 0000 UTC 29 Sep from the (b) CTRL and (c) DI runs initialized at 1200 UTC 28 Sep. The visible satellite image in (a) was obtained from the Naval Research Laboratory's Tropical Cyclone Page (https://www.nrlmry.navy.mil/tcdat/tc15/WPAC/21W.DUJUAN/vis/ols/2km).

and a small eye near the coastline. Although most of the cloud features were simulated in the CTRL run, the eye size was too large compared with that appearing in the satellite image, and the active rainband to the northnorthwest was considerably weaker than that in the observations (Fig. 9b). In the DI run (Fig. 9c), the inner-core structure was more axisymmetric, and the eye size was comparable with that in the satellite image. This is mainly attributed to the use of the vortex size adjustment in the new DI scheme. In addition, most of the rainbands were also reasonably well captured in the DI run.

\section{b. Typhoon Mujigae}

Typhoon Mujigae (2015) formed near Luzon Island in the Philippines and moved northwestward over the South China Sea toward southern China, with a lifetime peak intensity of MSW of $53.0 \mathrm{~m} \mathrm{~s}^{-1}$ and an MSLP of $944 \mathrm{hPa}$ according to the JTWC best-track data. Mujigae was affected by both of the islands of Luzon and Hainan during its short lifetime of about 3 days. The CTRL and DI runs were conducted with initial conditions from 0000 UTC 2 October to 1200 UTC 4 October at 12-h 

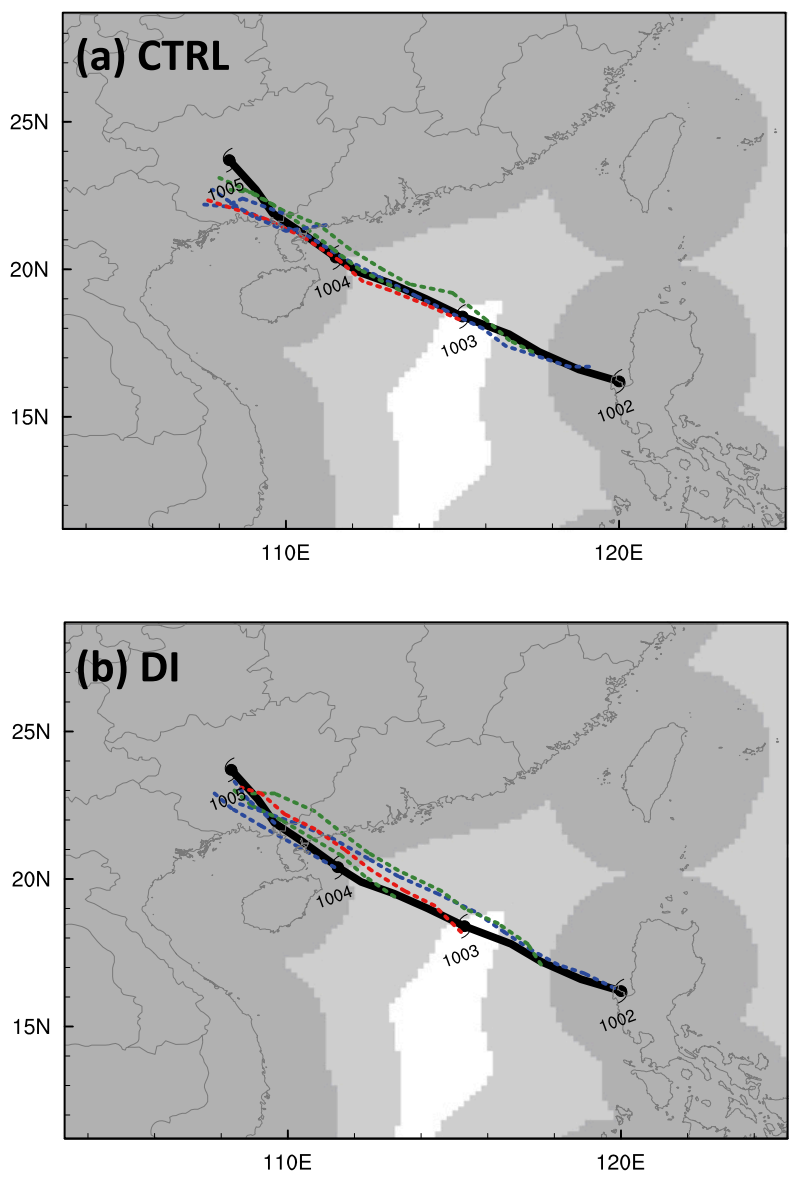

FIG. 10. As in Fig. 6, but for Typhoon Mujigae (2015) initialized from 0000 UTC 2 Oct to 1200 UTC 4 Oct with a 12-h interval. Red, green, and blue dashed lines are referred to as the STN1, STN2, and STN3 runs, respectively.

intervals and ended at 0000 UTC 5 October. The TC tracks from the CTRL and DI runs are compared with the JTWC best-track data in Fig. 10. Mujigae in both the CTRL and DI runs moved northwestward, similar to the JTWC best-track data, but the tracks in the DI runs showed a small northward bias. The initial mean position error was $41.4 \mathrm{~km}$ in the CTRL runs and $13.5 \mathrm{~km}$ in the DI runs. The latter was mainly due to some errors related to the interpolation of model variables from the intermediate mesh to the innermost mesh since the latter was not activated until the forecast runs. In the situations directly affected by terrain (STN2 and STN3), the mean 12-h position error in the hindcasts was $72.8 \mathrm{~km}$ in the CTRL runs and $58.2 \mathrm{~km}$ in the DI runs, and the storm weakened and dissipated after $12 \mathrm{~h}$. Note that the new DI scheme did not improve the TC track forecasts but reduced the initial position errors in this case compared to the CTRL runs. Nevertheless, in both the CTRL and DI runs, the track errors were not large in the Mujigae case.
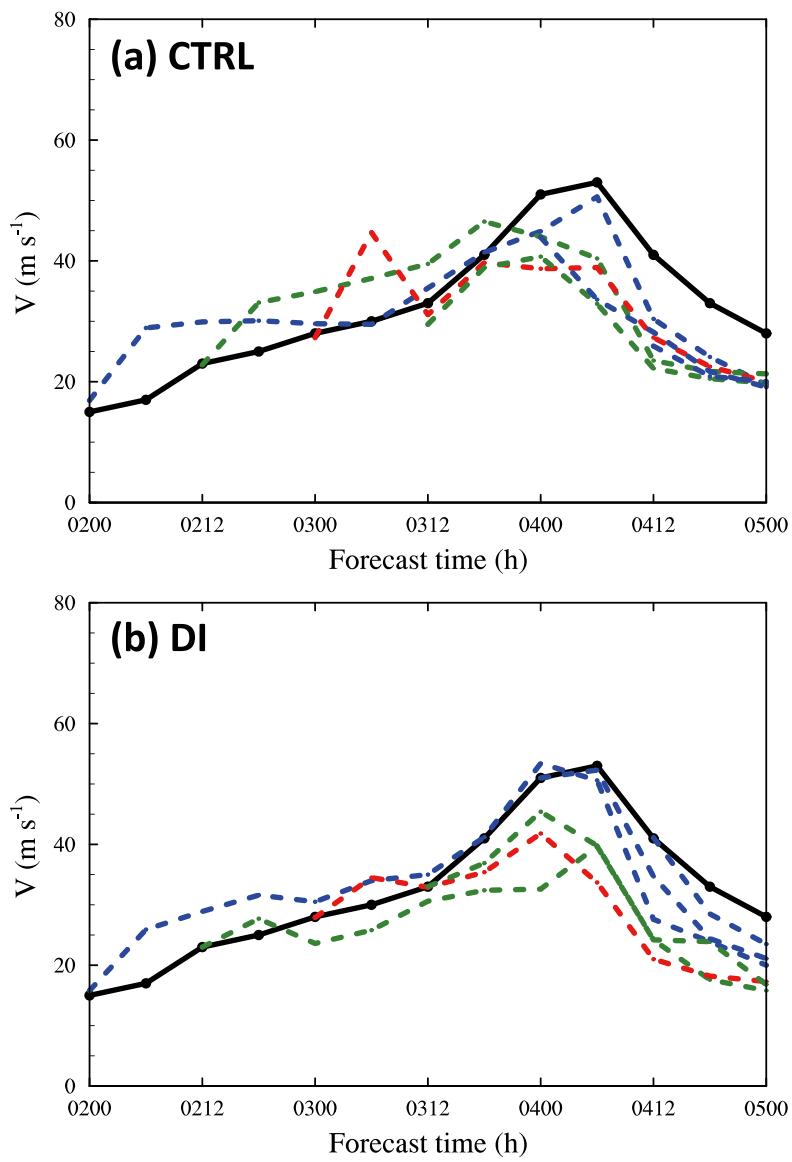

FIG. 11. As in Fig. 7, but for Typhoon Mujigae (2015). Red, green, and blue dashed lines are referred to as the STN1 STN2, and STN3 runs, respectively.

Figure 11 shows the 72-h intensity evolution in the JTWC best-track data and in both the CTRL and DI runs. The CTRL runs failed to capture the lifetime peak intensity except for one forecast initialized at 0000 UTC 2 October (Fig. 11a). With the new DI scheme, two forecasts captured well the lifetime peak intensity of Mujigae (Fig. 11b). The initial mean intensity error was $4.7 \mathrm{~m} \mathrm{~s}^{-1}$ in the CTRL runs and was only $0.2 \mathrm{~m} \mathrm{~s}^{-1}$ in the DI runs. For STN2 and STN3, the mean 12 -h intensity error was $8.9 \mathrm{~m} \mathrm{~s}^{-1}$ in the CTRL runs and $5.3 \mathrm{~m} \mathrm{~s}^{-1}$ in the DI runs. The results thus demonstrate that the new DI scheme largely reduced the initial TC intensity errors and also improved the subsequent intensity forecasts in the Mujigae case.

Figure 12 shows the mean sea level pressure and 10-m horizontal wind speed fields for the Mujigae case at the initial time of 0000 UTC 4 October when the storm was affected by both Hainan Island and the terrain around Guangdong Province. The MSLP was $994.1 \mathrm{hPa}$, and the MSW was $43.9 \mathrm{~m} \mathrm{~s}^{-1}$ in the CTRL run (Fig. 12a), while the MSLP and MSW in the JTWC best-track data were $948 \mathrm{hPa}$ and $51.0 \mathrm{~m} \mathrm{~s}^{-1}$, respectively. In the DI run 
(a)

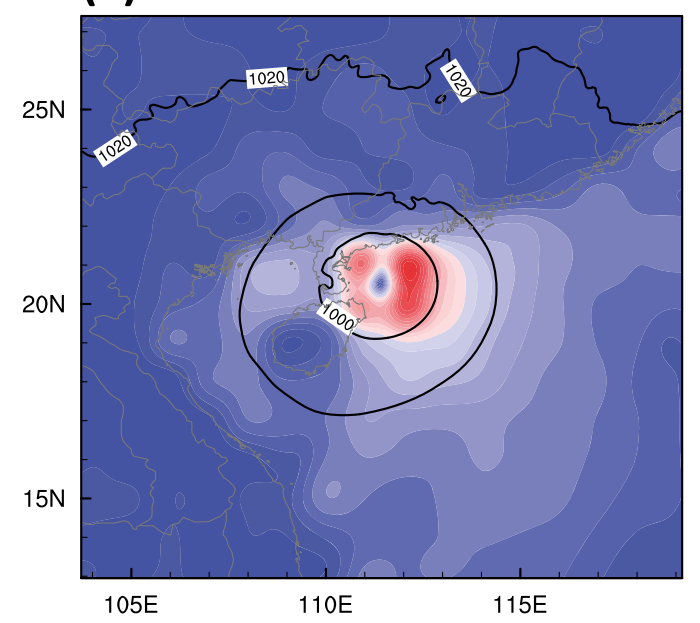

(b)

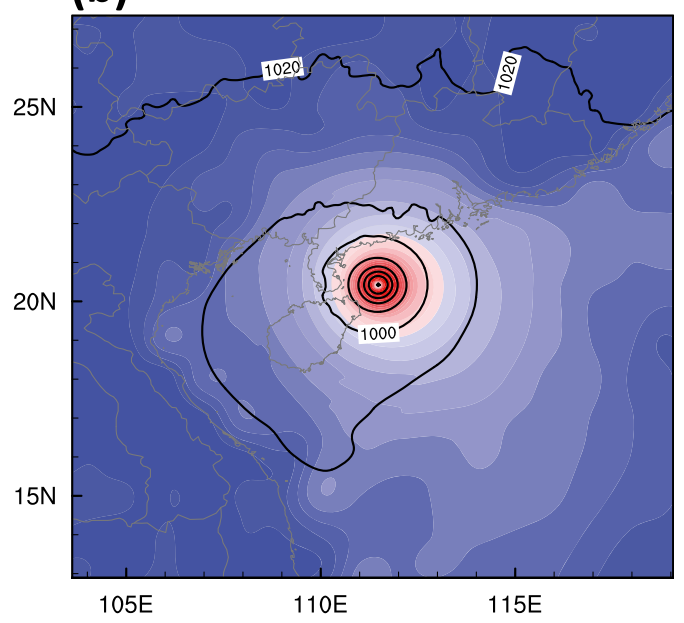

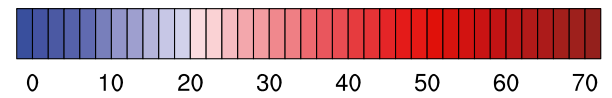

FIG. 12. As in Fig. 8, but for Typhoon Mujigae (2015) initialized at 0000 UTC 4 Oct.

(Fig. 12b), the vortex had an MSLP of $935.8 \mathrm{hPa}$ and the MSW was $51.0 \mathrm{~m} \mathrm{~s}^{-1}$, both of which were close to the values in the JTWC best-track dataset.

The radar reflectivity composited from the Doppler radars located at Zhanjiang (Z9759), Yangjiang (Z9662), Haikou (Z9898), Guangzhou (Z9200), and Sanya (Z9070) at 0600 UTC 4 October suggests that Mujigae had a complete eyewall with a very small eye and vigorous rainbands in the northeastern quadrant outside the inner core (Fig. 13a). The simulated maximum radar reflectivity in the CTRL run showed a too large eye (Fig. 13b) compared with that observed. There were two main rainbands around Mujigae at 0600 UTC 4 October: one connected with the eyewall to the south and that spiraled west-northwestward and the other to the north that spiraled east-southeastward. The CTRL run showed some similarities but with too large scales in both the azimuthal and radial directions compared with those observed. With the vortex size adjustment, the eye size in the DI run was much smaller than that in the CTRL run and was similar to that which appeared in the observed radar reflectivity except that the reflectivity in the eyewall and rainbands was slightly too high (Fig. 13c). Nevertheless, compared with the CTRL run, the DI run showed an overall improvement in the simulated inner-core structure and rainbands to some extent in the Mujigae case.

\section{Results for TCs affected by terrain over the western North Pacific in 2015}

To evaluate the overall performance of the new DI scheme, we conducted hindcasts for nine TCs that were affected by the terrain over the western North Pacific in 2015 (see Table 1), including Dujuan and Mujigae already discussed in section 4 . Since the new DI scheme treats three situations separately to spin up a TC vortex according to the distance between the TC center and the terrain as well as the height of the terrain, we evaluated the model performance for all hindcasts and also for hindcasts during the individual situations (Table 2). We also compared the track and intensity hindcasts with the official operational forecasts from JTWC (JTWC-fcst), China Meteorological Administration (CMA-fcst), and the NCEP operational Hurricane WRF Model (HWRFfcst). Note that the comparison here is only used for a rough reference because the initial TC position and intensity were based on the real-time data not the besttrack data in JTWC-fcst, CMA-fcst, and HWRF-fcst, and JTWC and CMA used different wind (1 vs 2 min) averages for storm intensity. In addition, the model settings between WRF and HWRF were different. Nevertheless, the comparison with JTWC-fcst, CMAfcst, and HWRF-fcst was attempted to demonstrate that the skill levels of these official or operational forecasts were all degraded for TCs under the influence of terrain and the use of our new DI scheme can improve those forecasts to some extent.

Figure 14 shows the absolute position and intensity errors for up to 72-h forecasts in the CTRL and DI runs and also from the three operational forecasts mentioned above. The mean initial position error for all forecasts was $50.5 \mathrm{~km}$, and the absolute position error decreased slightly during the first $12 \mathrm{~h}$, then increased with 

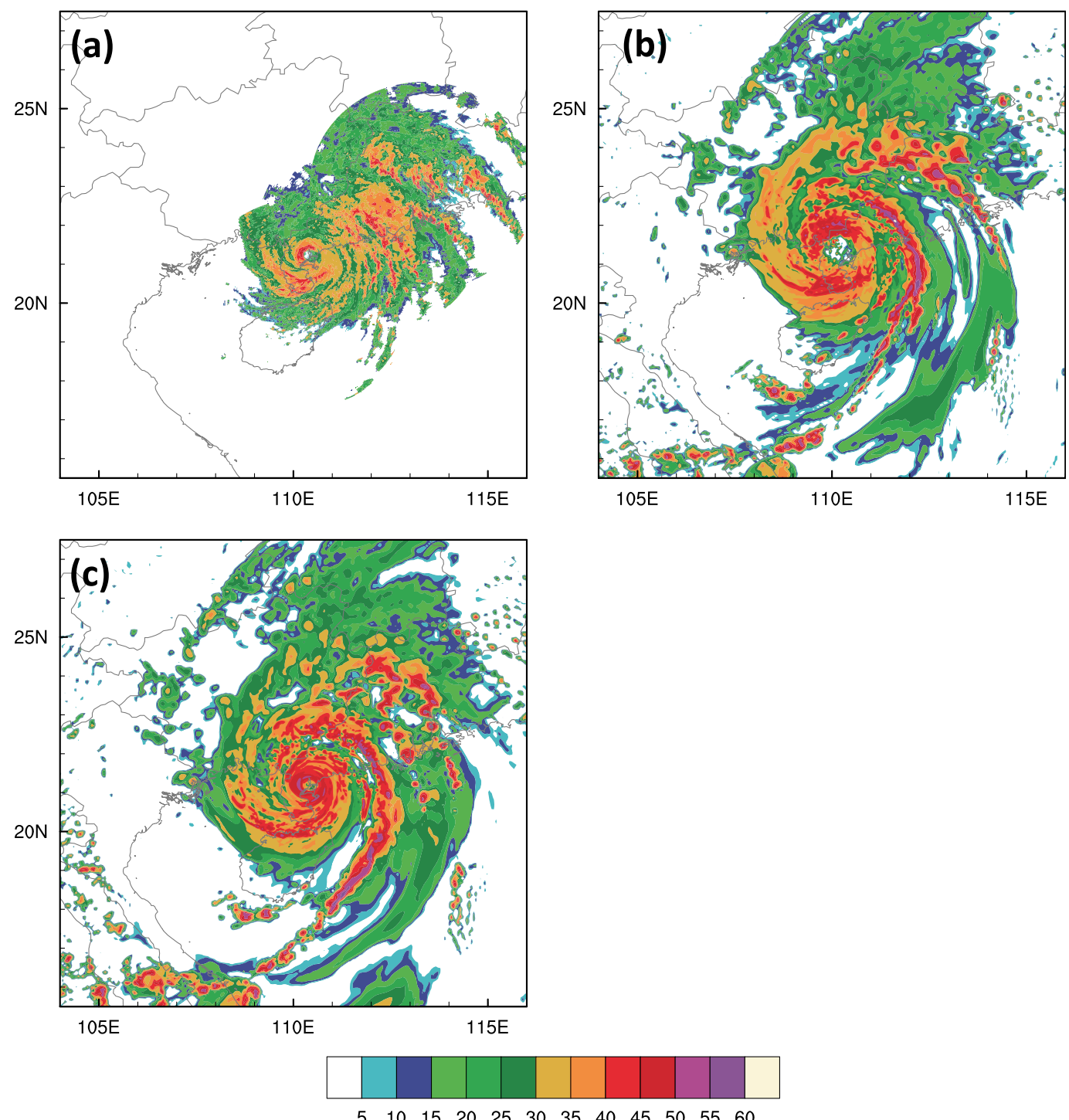

FIG. 13. Horizontal distributions of the (a) observed and modeled maximum radar reflectivities (dBZ) for Typhoon Mujigae (2015) at 0600 UTC 4 Oct 2015 in the (b) CTRL and (c) DI runs initialized at 0000 UTC 4 Oct 2015. The observations in (a) were composited from five Doppler radars located at Zhanjiang (Z9759), Yangjiang (Z9662), Haikou (Z9898), Guangzhou (Z9200), and Sanya (Z9070).

increasing forecast time afterward, and reached $218.6 \mathrm{~km}$ by $72 \mathrm{~h}$ in the CTRL runs. In the DI runs, the mean initial position error was $14.2 \mathrm{~km}$, and the position errors were smaller than those in the CTRL runs and HWRF-fcst from the 42- to 72-h forecasts but larger than JTWC-fcst and CMA-fcst. As a result, the mean position error for 72 -h forecasts was $203.0 \mathrm{~km}$ in the DI runs. This means that the new DI scheme reduced the initial position error by about $72 \%$ and the 72 -h position error by only $7 \%$ compared with the CTRL runs, but it showed slightly better performance than for the HWRF.
For the situation of STN1 (Fig. 14c), the initial position error was $50.0 \mathrm{~km}$ in the CTRL runs and $12.4 \mathrm{~km}$ in the DI runs. The 72 -h position error was $183.3 \mathrm{~km}$ in the CTRL runs and $185.9 \mathrm{~km}$ in the DI runs. Note that, for this situation, TCs were initially located over the open ocean, and the main difference between the CTRL and DI runs was the initial relocation of the TC vortex in the DI runs. As a result, the 72-h position errors were similar between the CTRL and DI runs. For the situations of STN2 (Fig. 14e) and STN3 (Fig. 14g), the position errors in the DI runs were smaller than those in other forecasts, especially at the initial time and after the $36-\mathrm{h}$ forecasts, indicating that 
TABLE 2. Members of TCs classified in the three situations in the new DI scheme.

\begin{tabular}{cll}
\hline \hline Situation & Member & \multicolumn{1}{c}{ Forecast initial time } \\
\hline STN1 & Noul & No. of forecasts \\
& Soudelor & 0000 UTC 5 May-0000 UTC 8 May \\
Dujuan & 1200 UTC 2 Aug-0000 UTC 7 Aug \\
Mujigae & 0000 UTC 24 Sep-1200 UTC 27 Sep \\
Koppu & 0000 UTC 3 Oct \\
Melor & 0000 UTC 15 Oct-1200 UTC 15 Oct \\
Mekkhala & 1200 UTC 12 Dec-0000 UTC 13 Dec \\
Noul & 1200 UTC 16 Jan \\
Linfa & 1200 UTC 8 May-1200 UTC 9 May \\
Mujigae & 1200 UTC 3 Jul-0000 UTC 4 Jul \\
& Koppu & 1200 UTC 2 Oct and 1200 UTC 3 Oct \\
Melor & 0000 UTC 15 Oct-0000 UTC 17 Oct \\
Mekkhala & 1200 UTC 13 Dec \\
Noul & 1200 UTC 17 Jan-0000 UTC 18 Jan \\
Kujira & 0000 UTC 10 May-1200 UTC 11 May \\
& Linfa & 0000 UTC 22 Jun-0000 UTC 23 Jun \\
& Soudelor & 1200 UTC 4 Jul-1200 UTC 6 Jul \\
& Dujuan & 1200 UTC 7 Aug - 0000 UTC 8 Aug \\
& Mujigae & 0000 UTC 28 Sep-1200 UTC 28 Sep \\
& Koppu & 0000 UTC 2 Oct and 0000-1200 UTC 4 Oct \\
Melor & 1200 UTC 17 Oct-1200 UTC 10 Oct \\
& 1200 UTC 14 Dec-0000 UTC 15 Dec \\
\hline
\end{tabular}

overall the new DI scheme improved the TC track forecast for TCs under the influence of the terrain. In addition, the 72-h position errors for STN2 and STN3 were larger than those for STN1, which was evidence of the difficulty in forecasting TCs that were affected directly by the terrain. The initial position errors were reduced by $51.5 \%$ and $75.5 \%$ for STN2 and STN3 in the DI runs, respectively, compared with those in the CTRL runs. The 72-h position errors were reduced by $25.0 \%$ and $40.6 \%$ for STN2 and STN3, respectively. Note that although a large reduction in the 72-h forecast position errors was achieved, the contribution to the mean absolute error for all cases was relatively small because of a very limited number of cases, especially for STN3 (Table 2).

The initial absolute intensity errors were 10.4 and $0.3 \mathrm{~m} \mathrm{~s}^{-1}$ in the CTRL and DI runs for the total cases (Fig. 14b). The absolute intensity error in the forecasts tended to decrease in the first $24 \mathrm{~h}$, then increased, and reached $11.9 \mathrm{~m} \mathrm{~s}^{-1}$ for the 72 -h forecasts in the CTRL runs. The absolute intensity errors tended to increase during all forecast times and reached $11.1 \mathrm{~m} \mathrm{~s}^{-1}$ for the 72-h forecasts in DI runs. The performance of the intensity forecast of HWRF-fcst was similar to that of the DI runs but the errors were larger in the first $6 \mathrm{~h}$ and after the $54-\mathrm{h}$ forecasts by HWRF-fcst. Both CMA-fcst and JTWC-fcst had smaller intensity errors than the CTRL and DI runs, as well as HWRF-fcst, except for the first 12-h forecasts.

For TCs initially over the open ocean (STN1; Fig. 14d), the mean absolute intensity errors were similar to those for all cases in both the CTRL and DI runs. The mean absolute initial intensity errors were 10.0 and $0.2 \mathrm{~m} \mathrm{~s}^{-1}$, and the $72-\mathrm{h}$ absolute intensity errors were 11.6 and $11.1 \mathrm{~m} \mathrm{~s}^{-1}$ in the CTRL and DI runs, respectively. The absolute intensity errors in the DI runs were smaller than those in the CTRL runs and in HWRF-fcst during the entire forecast time. For the situation of STN2 (Fig. 14f), the absolute initial intensity error was $0.2 \mathrm{~m} \mathrm{~s}^{-1}$ in the DI runs compared with $9.6 \mathrm{~m} \mathrm{~s}^{-1}$ in the CTRL runs. The absolute intensity errors in the first 36-h forecasts were also much smaller in the DI runs than in the CTRL runs. Note that the mean absolute initial intensity error was $2.4 \mathrm{~m} \mathrm{~s}^{-1}$ in HWRF-fcst, which used vortex relocation and initial intensity adjustment as well but was based on the real-time TC data not the besttrack data. The 72-h mean absolute intensity error was $11.1 \mathrm{~m} \mathrm{~s}^{-1}$ in the DI runs, which was slightly smaller than that in both the CTRL runs and HWRF forecasts. For the situation of STN3 (Fig. 14h), similar to the situation in STN2, the mean absolute intensity errors were systematically smaller in the DI runs than in the CTRL runs in the first 36-h forecasts and became similar afterward. Although the DI runs showed mean absolute intensity forecast errors similar to those for HWRF, the mean absolute initial intensity error was negligible in the DI runs but about $2.6 \mathrm{~m} \mathrm{~s}^{-1}$ in HWRF-fcst. This indicates that the new DI scheme can effectively remove the initial position and intensity errors and also improves TC track and intensity forecasts when TCs are directly affected by terrain.

Finally, since the new DI scheme includes both topographic adjustment and intensity/size adjustment, to isolate the contribution of topographic adjustment alone 
(a)

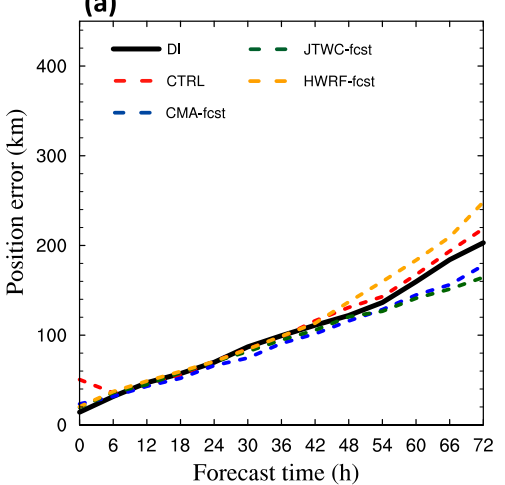

(c)

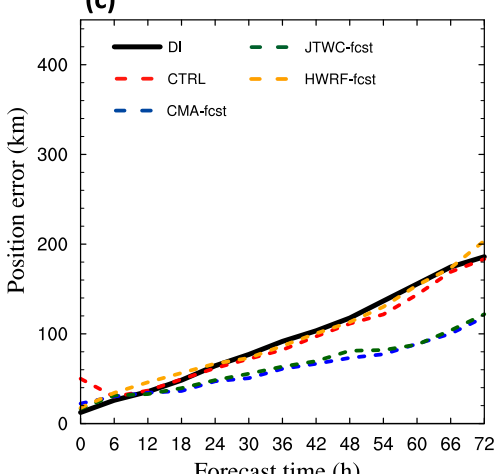

(e)

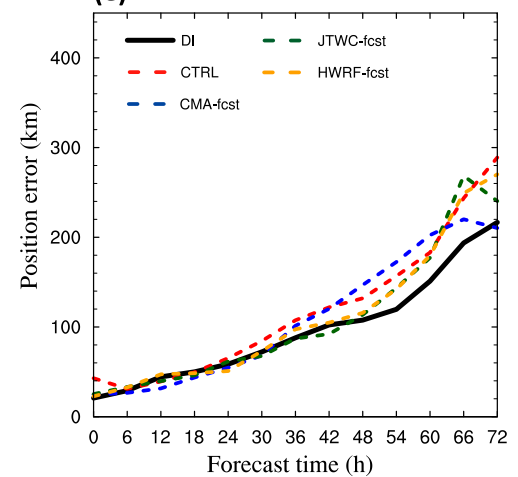

(g)

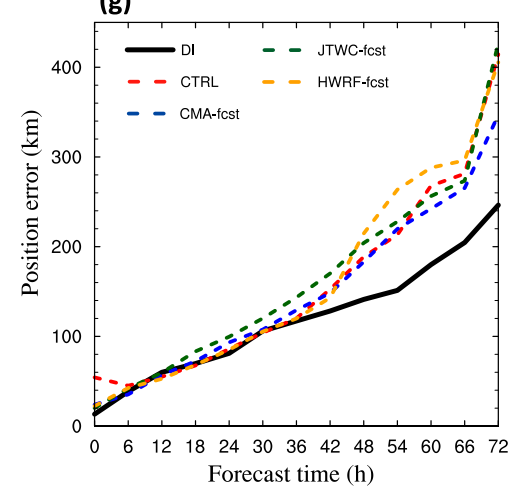

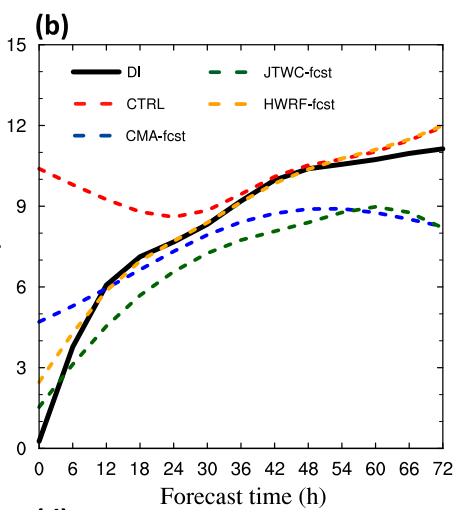
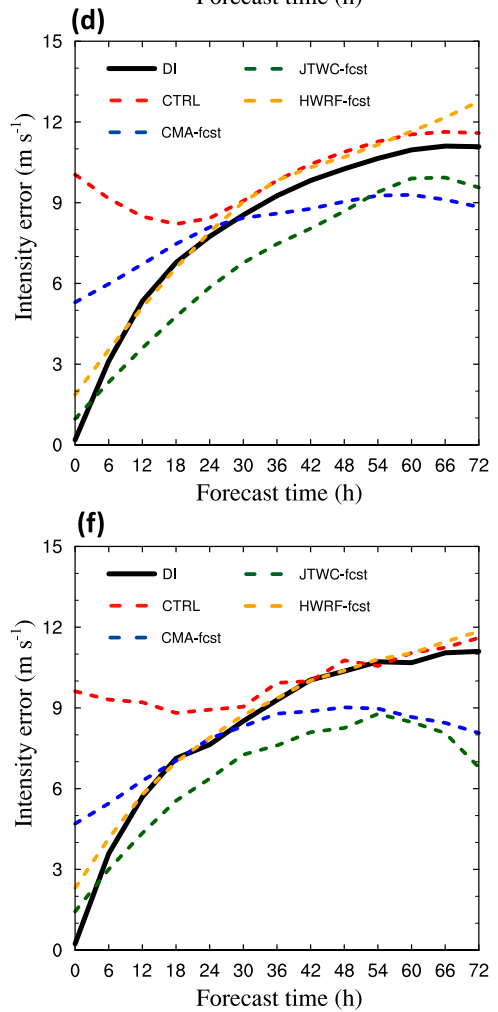

(h)

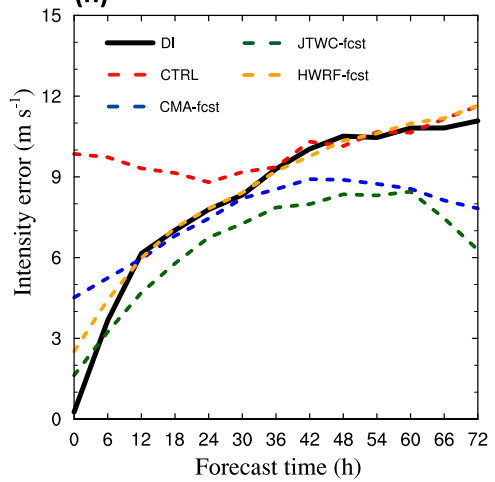

FIG. 14. Temporal evolution of (left) absolute position and (right) intensity errors for nine TCs affected by terrain over the western North Pacific during 2015 in the hindcasts from the CTRL (red dashed) and DI runs (black solid), and the official forecasts from CMA (blue dashed) and JTWC (green dashed), as well as from the HWRF Model (yellow dashed): (a),(b) all cases, (c),(d) STN1 cases, (e),(f) STN2 cases, and (g),(h) STN3 cases. 

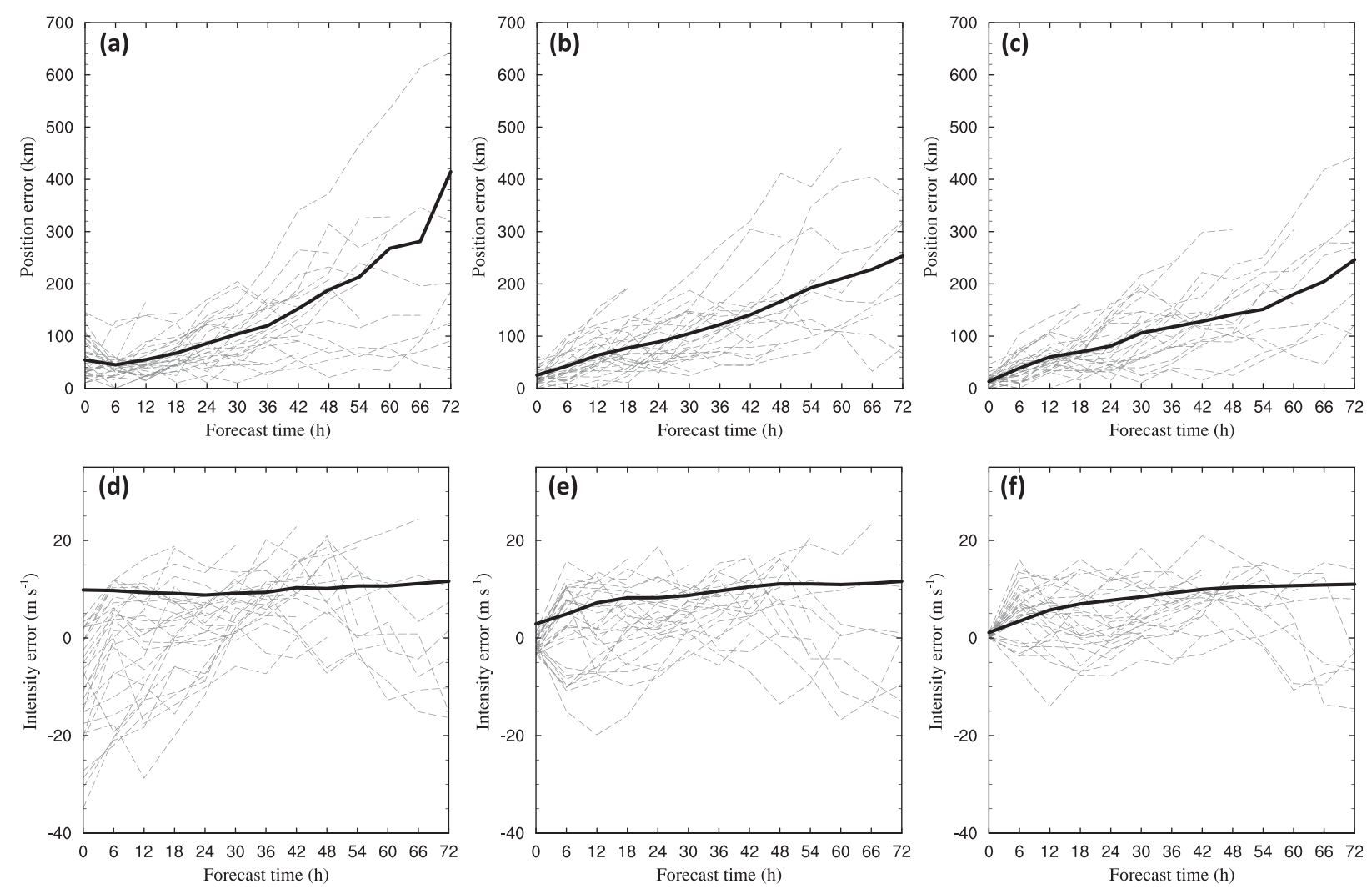

FIG. 15. Temporal evolution of (top) position and (bottom) intensity errors of TCs directly affected by terrain in STN3 (dashed) between the simulations and the JTWC best-track data and the average of the absolute errors (solid) at every 6h: (a),(d) CTRL runs, (b),(e) NOADJ runs, and (c),(f) DI runs.

to the improvements discussed above, hindcast experiments for the STN3 cases were rerun for DI and also for a new set of experiments using the new DI scheme without vortex intensity/size adjustment (NOADJ) with a cold model start for consistency (see section 3). Figure 15 shows the absolute position and intensity errors up to 72-h forecasts in the CTRL, DI, and NOADJ runs for all STN3 cases. Similar to the DI runs (Fig. 15c), the NOADJ runs reduced the absolute position errors during the whole forecast period (Fig. 15b) compared with CTRL (Fig. 15a), with the initial position error of $19.3 \mathrm{~km}$ and the 72 -h position error of $266.3 \mathrm{~km}$. The difference in the mean absolute position errors between the NOADJ and DI runs was generally small mainly because the TC motion was largely controlled by largescale environmental flow (Chan and Gray 1982). The mean initial intensity error was $2.2 \mathrm{~m} \mathrm{~s}^{-1}$ and the mean 72-h intensity error was $11.8 \mathrm{~m} \mathrm{~s}^{-1}$ in the NOADJ runs (Fig. 15e), both of which were smaller than those in the CTRL runs (Fig. 15d) but larger than those in the DI runs (Fig. 15f), suggesting that the intensity/size adjustment in the new DI scheme can reduce the intensity errors further, particularly the initial intensity errors.

\section{Summary}

In this study, the TC DI scheme based on cycle runs developed by Cha and Wang (2013) has been extended to the situations under the influence of terrain. Three situations are considered, including 1) over the open ocean, 2) near the low terrain, and 3) close to relatively high terrain or over low terrain inland. For TCs over the open ocean (STN1), the new DI steps are almost the same as those in Cha and Wang (2013) except for the use of a vortex size adjustment based on Liu and Tan (2016) and a vortex intensity adjustment before the forecast run. For TCs near low terrain (STN2), a special topographic component is introduced into the procedures in STN1. The topographic component includes two parts. One is defined as the difference between the WRF Model initial conditions interpolated from the global analysis data in pressure coordinates into the terrain-following hydrostatic-pressure vertical coordinate ( $\sigma$ coordinate) system with and without terrain. The other part includes the small-scale features (noise due to the presence of topography) with wavelengths less than $200 \mathrm{~km}$ as a residual from the orographic filtering algorithm. For TCs 
close to relatively high terrain or over low terrain inland (STN3), the cycle runs are conducted without the effects of terrain and land, namely, as over the open ocean, to spin up the axisymmetric component of the TC vortex, which is then merged with the environmental field in the initial conditions of the forecast run. Note that different from both STN1 and STN2, in which the forecast runs are warm started, STN3 is integrated with a cold start in its forecast runs since the cycle runs are conducted with no terrain.

Hindcasts for two cases-Supertyphoon Dujuan (2015) and Typhoon Mujigae (2015) - were analyzed in detail to demonstrate the strength of the new DI scheme. The results showed that the new DI scheme almost eliminated the initial position and intensity errors and also improved the subsequent intensity forecasts. The inner-core structure and the distribution of rainbands in the forecasts were more comparable with satellite and radar observations with the use of the new DI scheme than the forecasts in which no DI scheme was used.

Nine TCs affected by terrain over the western North Pacific in 2015 were selected to evaluate the overall performance of the new DI scheme. The initial position and absolute intensity errors were largely reduced in all DI runs (STN1, STN2, and STN3). For TCs directly affected by the terrain (STN2 and STN3), although the improvements in the track forecasts were relatively small, the intensity forecasts up to $36 \mathrm{~h}$ were considerably improved with the use of the new DI scheme. We also noticed that the current official TC forecasts and the high-resolution operational TC model HWRF also showed large forecast errors for TCs affected by terrain. As shown in Fig. 14, both track and intensity forecast errors from JTWC and CMA for the same nine TCs upon which we conducted hindcasts were increasingly large for TCs affected by terrain. We also showed that the performance of the WRF forecasts with our new DI scheme was comparable with that of the NCEP HWRF Model forecasts for the same nine storms. Additional experiments for TCs directly affected by terrain (STN3) demonstrated that the inclusion of the intensity/size adjustment in the new DI scheme also effectively contributed to the reduction of intensity errors, particularly the reduction of initial intensity errors.

Finally, we should point out that an obvious weakness of the new DI scheme developed in this study is its semiidealized cycle runs together with the cold model start for the situation of STN3, in which the strong interaction between the TC vortex and the terrain is not considered. Many previous studies have indicated that the complex interaction with the terrain may have various consequences, as mentioned in the introduction. Although better approaches that include the strong orographic interactions could be developed in future studies, the new DI scheme proposed in this study is currently practical and can be implemented easily in any numerical model. Therefore, it can be used to fill the gap where no effective scheme is currently available for TC DI under the influence of mesoscale and small-scale terrain, in particular for the western North Pacific, as shown in this study.

Acknowledgments. The authors are grateful to three anonymous reviewers for their constructive review comments and to Dr. Xinghai Zhang for providing the vortex intensity adjustment code used in our work. This study has been supported in part by the National Basic Research and Development Project (973 program) of China under Contract 2015CB452805 and in part by the National Natural Science Foundation of China under Grants 41575053, 41675044, and 41730960, as well as NSF Grant AGS-1326524 and the Basic Research Fund of CAMS (2016Z003). The official operational forecasts from JTWC and CMA were both obtained online (http://weather.unisys.com/hurricane/archive), as were the forecasts of the NCEP operational Hurricane WRF Model (http://www.emc.ncep.noaa.gov/gc_wmb/vxt/ HWRF), the FNL data (https://rda.ucar.edu/datasets/ ds083.2), and the JTWC best-track data (https://metoc. ndbc.noaa.gov/JTWC).

\section{REFERENCES}

Cha, D.-H., and Y. Wang, 2013: A dynamical initialization scheme for real-time forecasts of tropical cyclones using the WRF Model. Mon. Wea. Rev., 141, 964-986, https://doi.org/10.1175/ MWR-D-12-00077.1.

Chan, J. C. L., and W. M. Gray, 1982: Tropical cyclone movement and surrounding flow relationships. Mon. Wea. Rev., 110, 1354-1374, https://doi.org/10.1175/1520-0493(1982)110<1354: TCMASF $>2.0 . \mathrm{CO} ; 2$.

Chen, F., and J. Dudhia, 2001: Coupling an advanced land surfacehydrology model with the Penn State-NCAR MM5 modeling system. Part I: Model implementation and sensitivity. Mon. Wea. Rev., 129, 569-585, https://doi.org/10.1175/1520-0493 (2001) $129<0569$ :CAALSH $>2.0$. CO; 2 .

Davis, C., and Coauthors, 2008: Prediction of landfalling hurricanes with the Advanced Hurricane WRF Model. Mon. Wea. Rev., 136, 1990-2005, https://doi.org/10.1175/ 2007MWR2085.1.

Donelan, M. A., B. K. Haus, N. Reul, W. J. Plant, M. Stiassnie, H. C. Graber, O. B. Brown, and E. S. Saltzman, 2004: On the limiting aerodynamic roughness of the ocean in very strong winds. Geophys. Res. Lett., 31, L18306, https://doi.org/10.1029/ 2004 GL019460.

Dudhia, J., 1989: Numerical study of convection observed during the Winter Monsoon Experiment using a mesoscale twodimensional model. J. Atmos. Sci., 46, 3077-3107, https://doi. org/10.1175/1520-0469(1989)046<3077:NSOCOD>2.0.CO;2. 
Garratt, J. R., 1992: The Atmospheric Boundary Layer. Cambridge University Press, $316 \mathrm{pp}$.

Hendricks, E. A., M. S. Peng, and T. Li, 2013: Evaluation of multiple dynamic initialization schemes for tropical cyclone prediction. Mon. Wea. Rev., 141, 4028-4048, https://doi.org/ 10.1175/MWR-D-12-00329.1.

Holland, G. J., 1980: An analytic model of the wind and pressure profiles in hurricanes. Mon. Wea. Rev., 108, 1212-1218, https://doi. org/10.1175/1520-0493(1980)108<1212:AAMOTW>2.0.CO;2.

Hong, S. Y., J. Dudhia, and S. H. Chen, 2004: A revised approach to ice microphysical processes for the bulk parameterization of clouds and precipitation. Mon. Wea. Rev., 132, 103-120, https://doi.org/ 10.1175/1520-0493(2004)132<0103:ARATIM > 2.0.CO;2.

_- Y. Noh, and J. Dudhia, 2006: A new vertical diffusion package with an explicit treatment of entrainment processes. Mon. Wea. Rev., 134, 2318-2341, https://doi.org/10.1175/MWR3199.1.

Kain, J. S., and J. M. Fritsch, 1990: A one-dimensional entraining/ detraining plume model and its application in convective parameterization. J. Atmos. Sci., 47, 2784-2802, https://doi.org/ 10.1175/1520-0469(1990)047<2784:AODEPM >2.0.CO;2.

Kurihara, Y., M. A. Bender, and R. J. Ross, 1993: An initialization scheme of hurricane models by vortex specification. Mon. Wea. Rev., 121, 2030-2045, https://doi.org/10.1175/1520-0493 (1993) $121<2030$ :AISOHM>2.0.CO;2.

Kwon, I.-H., and H.-B. Cheong, 2010: Tropical cyclone initialization with a spherical high-order filter and an idealized threedimensional bogus vortex. Mon. Wea. Rev., 138, 1344-1367, https://doi.org/10.1175/2009MWR2943.1.

Lin, Y.-L., S.-Y. Chen, C. M. Hill, and C.-Y. Huang, 2005: Control parameters for the influence of a mesoscale mountain range on cyclone track continuity and deflection. J. Atmos. Sci., 62, 1849-1866, https://doi.org/10.1175/JAS3439.1.

—, N. C. Witcraft, and Y.-H. Kuo, 2006: Dynamics of track deflection associated with the passage of tropical cyclones over a mesoscale mountain. Mon. Wea. Rev., 134, 3509-3538, https:// doi.org/10.1175/MWR3263.1.

Liou, Y.-C., T.-C. C. Wang, and P.-Y. Huang, 2016: The inland eyewall reintensification of Typhoon Fanapi (2010) documented from an observational perspective using multipleDoppler radar and surface measurements. Mon. Wea. Rev., 144, 241-261, https://doi.org/10.1175/MWR-D-15-0136.1.

Liu, H.-Y., and Z.-M. Tan, 2016: A dynamical initialization scheme for binary tropical cyclones. Mon. Wea. Rev., 144, 4787-4803, https://doi.org/10.1175/MWR-D-16-0176.1.

Mlawer, E. J., S. J. Taubman, P. D. Brown, M. J. Iacono, and S. A. Clough, 1997: Radiative transfer for inhomogeneous atmospheres: RRTM, a validated correlated-k model for the longwave. J. Geophys. Res., 102, 16 663-16 682, https://doi.org/ 10.1029/97JD00237.
Nguyen, H. V., and Y.-L. Chen, 2011: High-resolution initialization and simulations of Typhoon Morakot (2009). Mon. Wea. Rev., 139, 1463-1491, https://doi.org/10.1175/2011MWR3505.1.

Ramsay, H. A., and L. M. Leslie, 2008: The effects of complex terrain on severe landfalling Tropical Cyclone Larry (2006) over northeast Australia. Mon. Wea. Rev., 136, 4334-4354, https://doi.org/10.1175/2008MWR2429.1.

Rappin, E. D., D. S. Nolan, and S. J. Majumdar, 2013: A highly configurable vortex initialization method for tropical cyclones. Mon. Wea. Rev., 141, 3556-3575, https://doi.org/10.1175/ MWR-D-12-00266.1.

Shieh, S. L., S. T. Wang, M. D. Cheng, and T. C. Yeh, 1998: Tropical cyclone tracks over Taiwan and its vicinity for the one hundred years from 1987 to 1996 (in Chinese). Central Weather Bureau Research Rep. CWB86-1M-01, 497 pp.

Skamarock, W. C., and Coauthors, 2008: A description of the Advanced Research WRF version 3. NCAR Tech. Note NCAR/ TN-475+STR, 113 pp., http://dx.doi.org/10.5065/D68S4MVH.

Wang, S. T., 1980: Prediction of the behavior and intensity of typhoons in Taiwan and its vicinity (in Chinese). Chinese National Science Council Research Rep. 108, 100 pp.

Wu, C.-C., 2001: Numerical simulation of Typhoon Gladys (1994) and its interaction with Taiwan terrain using the GFDL hurricane model. Mon. Wea. Rev., 129, 1533-1549, https://doi.org/ 10.1175/1520-0493(2001)129<1533:NSOTGA $>2.0 . C O ; 2$.

Yang, M.-J., D.-L. Zhang, and H.-L. Huang, 2008: A modeling study of Typhoon Nari (2001) at landfall. Part I: Topographic effects. J. Atmos. Sci., 65, 3095-3115, https://doi.org/10.1175/ 2008JAS2453.1.

Yeh, T.-C., and R. L. Elsberry, 1993: Interaction of typhoons with the Taiwan topography. Part II: Continuous and discontinuous tracks across the island. Mon. Wea. Rev., 121, 3213-3233, https://doi.org/10.1175/1520-0493(1993) 121<3213:IOTWTT>2.0.CO;2.

— L.-F. Hsiao, D.-S. Chen, and K.-N. Huang, 2012: A study on terrain-induced tropical cyclone looping in east Taiwan: Case study of Typhoon Haitang in 2005. Nat. Hazards, 63, 1497-1514, https://doi.org/10.1007/s11069-011-9876-7.

Yu, C.-K., and C.-L. Tsai, 2010: Surface pressure features of landfalling typhoon rainbands and their possible causes. J. Atmos. Sci., 67, 2893-2911, https://doi.org/10.1175/2010JAS3312.1.

Zhang, S., T. Li, X. Ge, M. Peng, and N. Pan, 2012: A 3DVARbased dynamical initialization scheme for tropical cyclone predictions. Wea. Forecasting, 27, 473-483, https://doi.org/ 10.1175/WAF-D-10-05066.1.

Zou, X., and Q. Xiao, 2000: Studies on the initialization and simulation of a mature hurricane using a variational bogus data assimilation scheme. J. Atmos. Sci., 57, 836-860, https://doi. org/10.1175/1520-0469(2000)057<0836:SOTIAS >2.0.CO;2. 